MATHEMATICS OF COMPUTATION

Volume 73, Number 247, Pages 1167-1193

S 0025-5718(04)01634-5

Article electronically published on January 23, 2004

\title{
AN ADAPTIVE FINITE ELEMENT ALGORITHM WITH RELIABLE AND EFFICIENT ERROR CONTROL FOR LINEAR PARABOLIC PROBLEMS
}

\author{
ZHIMING CHEN AND JIA FENG
}

\begin{abstract}
An efficient and reliable a posteriori error estimate is derived for linear parabolic equations which does not depend on any regularity assumption on the underlying elliptic operator. An adaptive algorithm with variable time-step sizes and space meshes is proposed and studied which, at each time step, delays the mesh coarsening until the final iteration of the adaptive procedure, allowing only mesh and time-step size refinements before. It is proved that at each time step the adaptive algorithm is able to reduce the error indicators (and thus the error) below any given tolerance within a finite number of iteration steps. The key ingredient in the analysis is a new coarsening strategy. Numerical results are presented to show the competitive behavior of the proposed adaptive algorithm.
\end{abstract}

\section{INTRODUCTION}

A posteriori error estimates are computable quantities in terms of the discrete solution and data that measure the actual discrete errors without the knowledge of exact solutions. They are essential in designing algorithms for mesh and time-step size modifications which equidistribute the computational effort and optimize the computation. Ever since the pioneering work of Babuška and Rheinboldt [4], the adaptive finite element methods based on a posteriori error estimates have become a central theme in scientific and engineering computations. There are considerable efforts in the literature devoted to the development of efficient adaptive algorithms for various linear and nonlinear parabolic partial differential equations. In particular, a posteriori error estimates are derived in Bieterman and Babuška [2], [3] and Moore [14 for one-dimensional and in Eriksson and Johnson [12, 13, Picasso [17], and Verfürth [20 for multidimensional linear and mildly nonlinear parabolic problems. Efficient adaptive procedures based on a posteriori error estimates are also developed in Chen and Dai [5], Chen, Nochetto and Schmidt 7], Nochetto, Schmidt and Verdi [16] for solving nonlinear partial differential equations arising from physical and industrial processes.

Received by the editor September 28, 2001 and, in revised form, January 12, 2003.

2000 Mathematics Subject Classification. Primary 65N15, 65N30, 65N50.

The first author was supported in part by China NSF under the grant 10025102 and by China MOS under the grant G1999032802. 
Let $\Omega$ be a polyhedron domain in $\mathbf{R}^{d}(d=1,2,3), \Gamma=\partial \Omega$, and $T>0$. We consider the following linear parabolic equation:

$$
\begin{array}{ll}
\frac{\partial u}{\partial t}-\operatorname{div}(a(x) \nabla u)=f & \text { in } \Omega \times(0, T), \\
u=0 \quad \text { on } \Gamma \times(0, T), & u(x, 0)=u_{0}(x) \quad \text { in } \Omega,
\end{array}
$$

where $f \in L^{2}\left(0, T ; L^{2}(\Omega)\right), u_{0} \in L^{2}(\Omega)$, and $a(x)$ is a piecewise constant function. Let $\tau_{n}$ be the $n$-th time-step size and $t^{n}=\sum_{i=1}^{n} \tau_{i}$. Denote by $\mathcal{M}^{n}$ a uniformly regular partition of $\Omega$ into simplexes such that $a(x)$ is a constant on each element $K \in \mathcal{M}^{n}$. We use refinement/coarsening procedures based on the bisection algorithm (cf., e.g., Bänsch [1]), which lead to compatible consecutive meshes whose minimum angles are bounded uniformly away from zero. Two meshes are compatible if one is the local refinement by bisection of the other. Let $V^{n}$ indicate the usual space of conforming linear finite elements over $\mathcal{M}^{n}$ and $V_{0}^{n}=V^{n} \cap H_{0}^{1}(\Omega)$. Let $U_{h}^{0}=P_{0} u_{0}$, where $P_{0}: L^{2}(\Omega) \rightarrow V_{0}^{0}$ is the $L^{2}$ projection operator into the linear finite element space $V_{0}^{0}$ over the initial mesh $\mathcal{M}^{0}$. We consider the following simple fully discrete finite element scheme for solving (1.1). For $n=1,2, \ldots$, find $U_{h}^{n} \in V_{0}^{n}$ such that

$$
\left\langle\frac{U_{h}^{n}-U_{h}^{n-1}}{\tau_{n}}, v\right\rangle+\left\langle a \nabla U_{h}^{n}, \nabla v\right\rangle=\left\langle\bar{f}^{n}, v\right\rangle \quad \forall v \in V_{0}^{n},
$$

where $\langle\cdot, \cdot\rangle$ stands for the inner product on $L^{2}(\Omega)$, and $\bar{f}^{n}=\frac{1}{\tau_{n}} \int_{t^{n-1}}^{t^{n}} f(x, t) d t$.

The main tool in deriving a posteriori error estimates in [5], 7], 12], 13], [16] is the analysis of linear dual problems of the corresponding error equations. The derived a posteriori error estimates, however, depend on the $H^{2}$ regularity assumption on the underlying elliptic operator. Without using this regularity assumption, the energy method is used in [17] to derive an a posteriori error estimate for the total energy error of the approximate solution. A lower bound for the local error is also derived for the associated a posteriori error indicator in that paper. We remark that while the energy method applies to less regular solutions than the duality method, the latter yields a better rate in the case of regular solutions. Also the norms that the error is being measured in are different. The duality technique applies to lower order norms and yields better results for them when the underlying elliptic operators are regular.

The results in 17 are obtained under the conditions that (1) the space-time mesh $\left\{\left(t^{n-1}, t^{n}\right) \times K: K \in \mathcal{M}^{n}\right\}$ is regular in the sense of [9], and (2) the finite element spaces are nested $V^{n-1} \subset V^{n}$; that is, no mesh coarsening is allowed. In this paper, without these two conditions we derive an a posteriori error estimate for the total energy error based on a direct energy estimate argument which has been used in Chen, Nochetto and Schmidt [8] for the phase relaxation model, a system of one parabolic equation coupled with one variational inequality. This energy estimate argument is slightly different from that of [17]. We also prove a lower bound for the local error for our a posteriori error indicator without using the space-mesh regularity assumption in [17].

Let $U^{n} \in H_{0}^{1}(\Omega)$ be the solution of the following semidiscrete problem

$$
\left\langle\frac{U^{n}-U^{n-1}}{\tau_{n}}, \varphi\right\rangle+\left\langle a \nabla U^{n}, \nabla \varphi\right\rangle=\left\langle\bar{f}^{n}, \varphi\right\rangle \quad \forall \varphi \in H_{0}^{1}(\Omega) .
$$


We observe that by modifying the time-step size $\tau_{n}$, we are essentially controlling the error between $u^{n}=u\left(x, t^{n}\right)$ and $U^{n}$, not between $u^{n}$ and $U_{h}^{n}$. Moreover, if we let $U_{*}^{n} \in H_{0}^{1}(\Omega)$ be the solution of the auxiliary problem

$$
\left\langle\frac{U_{*}^{n}-U_{h}^{n-1}}{\tau_{n}}, \varphi\right\rangle+\left\langle a \nabla U_{*}^{n}, \nabla \varphi\right\rangle=\left\langle\bar{f}^{n}, \varphi\right\rangle \quad \forall \varphi \in H_{0}^{1}(\Omega)
$$

then we also observe that for fixed time-step size $\tau_{n}$, by adapting the mesh $\mathcal{M}^{n}$ we are essentially controlling the error between $U_{h}^{n}$ and $U_{*}^{n}$, not between $U_{h}^{n}$ and $U^{n}$ (or the exact solution $u$ ). These two observations play an important role in the subsequent analysis to prove the lower bound for the space a posteriori error indicator and in the development of the refinement/coarsening strategy.

Based on the local error indicators, the usual adaptive algorithm solving the parabolic problem (1.1) at the $n$-th time step reads as

$$
\text { Solve } \rightarrow \text { Estimate } \rightarrow \text { Refine/Coarsen. }
$$

Here the refinement/coarsening procedure includes both the mesh and time-step size modifications. There are several possibilities proposed in the literature on the strategies of the adaptive control of meshes and time-step sizes. We refer to Schmidt and Siebert [18] for a nice review of various adaptive algorithms and their implementation details. In this paper, at each time step $n$, we propose the following algorithm to modify the time-step size $\tau_{n}$ and mesh $\mathcal{M}^{n}$ starting from the initial time-step size $\tau_{n, 0}=\tau_{n-1}$ and initial mesh $\mathcal{M}^{n, 0}=\mathcal{M}^{n-1}$ :

1. Refine the time-step size $\tau_{n .0}$ and the mesh $\mathcal{M}^{n, 0}$ so that for the solution $U_{h}^{n} \in V_{0}^{n}$ of (1.2) on the final mesh $\mathcal{M}^{n}$ with final time-step size $\tau_{n}$, the associated space and time error indicators are less than the prescribed tolerances respectively;

2. Coarsen the mesh $\mathcal{M}^{n}$ so that for the solution $U_{H}^{n}$ on the coarsened mesh $\mathcal{M}_{H}^{n}$, the associated coarsening error indicator is less than some prescribed tolerance;

3. Enlarge the initial time-step size $\tau_{n+1,0}$ for next time step if the current time error indicator is much less than the tolerance.

We will extend the convergence analysis of adaptive finite element methods developed for linear elliptic problems in Dörfler [11] and Morin, Nochetto and Siebert [15] to prove that the iteration in Step 1 of the above algorithm terminates in a finite number of steps. The main difficulty now is the treatment of the oscillation of the residual $R^{n}=\bar{f}^{n}-\left(U_{h}^{n}-U_{h}^{n-1}\right) / \tau_{n}$ which changes at each refinement procedure. The oscillation of any function $\varphi \in L^{2}(\Omega)$ over the mesh $\mathcal{M}^{n}$ is defined as

$$
\operatorname{osc}\left(\varphi, \mathcal{M}^{n}\right)=\left(\sum_{K \in \mathcal{M}^{n}} h_{K}^{2}\left\|\varphi-P_{K} \varphi\right\|_{L^{2}(K)}^{2}\right)^{1 / 2}
$$

where $P_{K} \varphi=\frac{1}{|K|} \int_{K} \varphi d x$. For fixed time step size $\tau_{n}$, let $\mathcal{M}^{n, k+1}$ be a refinement of the mesh $\mathcal{M}^{n, k}$, and $U_{k}^{n, k+1}, U_{h}^{n, k}$ be the corresponding solutions of (1.2) over the meshes $\mathcal{M}^{n, k+1}, \mathcal{M}^{n, k}$. Note that since $R^{n, k+1} \neq R^{n, k}$, the following relation, which is crucial in the argument in [15], is no longer valid:

$$
\operatorname{osc}\left(R^{n, k+1}, \mathcal{M}^{n, k+1}\right)^{2} \leq \zeta \operatorname{osc}\left(R^{n, k}, \mathcal{M}^{n, k}\right)^{2}, \text { for some constant } \zeta<1 .
$$


Instead, we show in Lemma 4.5 that

$$
\operatorname{osc}\left(R^{n, k+1}, \mathcal{M}^{n, k+1}\right)^{2} \leq \zeta_{1} \operatorname{osc}\left(R^{n, k}, \mathcal{M}^{n, k}\right)^{2}+\zeta_{2}\left\|U_{h}^{n, k+1}-U_{h}^{n, k}\right\|_{\tau_{n}, \Omega}^{2}
$$

for some constants $0<\zeta_{1}<1$ and $\zeta_{2}>0$. Here $\|\cdot\|_{\tau_{n}, \Omega}$ is the weighted norm of $H^{1}(\Omega)$ with parameter $\tau_{n}>0$,

$$
\|\varphi\|_{\tau_{n}, \Omega}=\left(\frac{1}{\tau_{n}}\|\varphi\|_{L^{2}(\Omega)}^{2}+\|\varphi\|_{\Omega}^{2}\right)^{1 / 2} \quad \forall \varphi \in H^{1}(\Omega)
$$

with $\|\varphi\|_{\Omega}=\langle a \nabla \varphi, \nabla \varphi\rangle^{1 / 2}$, the energy seminorm of $\varphi$. The additional term $\left\|U_{h}^{n, k+1}-U_{h}^{n, k}\right\|_{\tau_{n}, \Omega}^{2}$ in (1.6) can be controlled by extending the technique in [15].

The choice of the coarsening error indicator and coarsening strategy in Step 2 is a subtle issue. The error incurred due to the over-coarsening can only be reduced by re-refining the coarsened mesh. Thus over-coarsening leads to the unnecessary solution of discrete problems, that is usually expensive and undesirable. Our coarsening error indicator is based on a direct control of the error between the coarsened discrete solution and the continuous solution $U_{*}^{n}$ of (1.4). More precisely, let $\mathcal{M}_{H}^{n}$ be a coarsening of $\mathcal{M}^{n}$, and $U_{H}^{n}, U_{h}^{n}$ be the corresponding solutions of (1.2) with fixed time-step size $\tau_{n}$. Since $V_{0}^{n, H} \subset V_{0}^{n}$, we deduce by Galerkin orthogonality (see Theorem 3.1 below) that

$$
\left\|U_{*}^{n}-U_{H}^{n}\right\|_{\tau_{n}, \Omega}^{2} \leq\left\|U_{*}^{n}-U_{h}^{h}\right\|_{\tau_{n}, \Omega}^{2}+\left\|U_{h}^{n}-I_{H}^{n} U_{h}^{n}\right\|_{\tau_{n}, \Omega}^{2},
$$

where $I_{H}^{n}: C(\bar{\Omega}) \rightarrow V^{n, H}$ is the usual linear finite element interpolant over $\mathcal{M}_{H}^{n}$. Our coarsening error indicator is then given by

$$
\eta_{\text {coarse }}^{n}:=\frac{1}{\tau_{n}}\left\|U_{h}^{n}-I_{H}^{n} U_{h}^{n}\right\|_{L^{2}(\Omega)}^{2}+\left\|U_{h}^{n}-I_{H}^{n} U_{h}^{n}\right\|_{\Omega}^{2},
$$

which does not depend on the coarsened solution $U_{H}^{n}$. We will show that this direct error control leads to only one coarsening step.

The rest of the paper is organized as follows: In $\S 2$ we derive an a posteriori error estimate between the solution $U_{h}^{n}$ of the discrete problem (1.2) and the solution $u$ of the continuous problem (1.1). A lower bound for the local error in terms of our space error indicator will be established which indicates that over-refinement will not occur for the refinement procedure based on this indicator. In $\S 3$ we introduce our adaptive algorithm and justify its various steps. In $\S 4$ we prove that at each time step our adaptive algorithms are able to reduce the error indicators below any given tolerance within a finite number of iterations. In $\S 5$ we discuss the coarsening algorithm and present one numerical example to show the competitive behavior of the proposed adaptive algorithm.

\section{A POSTERIORI ERROR ANALYSIS}

We begin with introducing some notation. For any open set $G \subset \mathbf{R}^{d}$, we denote by $H^{1}(G)$ the standard Sobolev space of functions in $L^{2}(G)$ whose first derivatives are also in $L^{2}(G)$. The coefficient $a(x)$ is assumed to be piecewise constant and positive. Thus the seminorm $\|\cdot\|_{G}$ defined by $\|\varphi\|_{G}^{2}=\langle a \nabla \varphi, \nabla \varphi\rangle_{G}$ is equivalent to the $H_{0}^{1}(\Omega)$ norm when $G=\Omega$. Here $\langle\cdot, \cdot\rangle_{G}$ stands for the $L^{2}(G)$ inner product or the duality pair between $H^{-1}(G)$ and $H_{0}^{1}(G)$. Throughout we write $\langle\cdot, \cdot\rangle=\langle\cdot, \cdot\rangle_{\Omega}$.

Given $f \in L^{2}\left(0, T ; L^{2}(\Omega)\right)$ and $u_{0} \in L^{2}(\Omega)$, the weak formulation of (1.1) reads as follows. Find $u \in L^{2}\left(0, T ; H_{0}^{1}(\Omega)\right) \cap H^{1}\left(0, T ; H^{-1}(\Omega)\right)$ such that $u(\cdot, 0)=u_{0}(\cdot)$, 
and for a.e. $t \in(0, T)$ the relation

$$
\left\langle\frac{\partial u}{\partial t}, \varphi\right\rangle+\langle a \nabla u, \nabla \varphi\rangle=\langle f, \varphi\rangle \quad \forall \varphi \in H_{0}^{1}(\Omega)
$$

holds.

In this section we consider the a posteriori error analysis for the full time-space discretization in $\S 2.1$. The lower bound of the local error in terms of the space error indicator is derived in $\S 2.2$.

2.1. The upper bound. We introduce the implicit Euler scheme for solving (2.1). Let $\tau_{n}$ be the $n$-th time-step size and set

$$
t^{n}:=\sum_{i=1}^{n} \tau_{i}, \quad \varphi^{n}(\cdot)=\varphi\left(\cdot, t^{n}\right)
$$

for any function $\varphi$ continuous in $\left(t^{n-1}, t^{n}\right]$. Let $N$ be the total number of steps; that is $t^{N} \geq T$. At each time step $n, n=1,2, \ldots, N$, we denote by $\mathcal{M}^{n}$ a uniformly regular partition of $\Omega$ into simplexes which is obtained from $\mathcal{M}^{n-1}$ by using refinement/coarsening procedures. Given an element $K \in \mathcal{M}^{n}, h_{K}$ stands for its diameter. Denote by $\mathcal{B}^{n}$ the collection of interior interelement sides $e$ of $\mathcal{M}^{n}$ in $\Omega$; $h_{e}$ stands for the size of $e \in \mathcal{B}^{n}$. Let $V^{n}$ indicate the usual space of conforming linear finite elements over $\mathcal{M}^{n}$, and $V_{0}^{n}=V^{n} \cap H_{0}^{1}(\Omega)$. Let $U_{h}^{0}=P_{0} u_{0}$, where $P_{0}: L^{2}(\Omega) \rightarrow V_{0}^{0}$ is the $L^{2}$ projection operator into the linear finite element space $V_{0}^{0}$ over the initial mesh $\mathcal{M}^{0}$. Then the fully discrete finite element approximation at the $n$-th time step reads as follows. Given $U_{h}^{n-1} \in V_{0}^{n-1}$, then $\mathcal{M}^{n-1}$ and $\tau_{n-1}$ are modified as described below to give rise to $\mathcal{M}^{n}$ and $\tau_{n}$ and, thereafter, $U_{h}^{n} \in V_{0}^{n}$ computed according to the following prescription:

$$
\left\langle\frac{U_{h}^{n}-U_{h}^{n-1}}{\tau_{n}}, v\right\rangle+\left\langle a \nabla U_{h}^{n}, \nabla v\right\rangle=\left\langle\bar{f}^{n}, v\right\rangle \quad \forall v \in V_{0}^{n} .
$$

We define the interior residual

$$
R^{n}:=\bar{f}^{n}-\frac{U_{h}^{n}-U_{h}^{n-1}}{\tau_{n}},
$$

along with the jump residual across $e \in \mathcal{B}^{n}$

$$
J_{e}^{n}:=\llbracket a \nabla U_{h}^{n} \rrbracket_{e} \cdot \nu_{e}=\left(\left.a \nabla U_{h}^{n}\right|_{K_{1}}-\left.a \nabla U_{h}^{n}\right|_{K_{2}}\right) \cdot \nu_{e}, \quad e=\partial K_{1} \cap \partial K_{2},
$$

using the convention that the unit normal vector $\nu_{e}$ to $e$ points from $K_{2}$ to $K_{1}$. We observe that integration by parts implies

$$
\left\langle a \nabla U_{h}^{n}, \nabla \varphi\right\rangle=-\sum_{e \in \mathcal{B}^{n}} \int_{e} J_{e}^{n} \varphi d s \quad \forall \varphi \in H_{0}^{1}(\Omega) .
$$

Theorem 2.1. For any integer $1 \leq m \leq N$, there exists a constant $C>0$ depending only on the minimum angle of meshes $\mathcal{M}^{n}, n=1,2, \ldots, m$, and the coefficient 
$a(x)$ such that the a posteriori error estimate

$$
\begin{aligned}
& \frac{1}{2}\left\|u^{m}-U_{h}^{m}\right\|_{L^{2}(\Omega)}^{2}+\sum_{n=1}^{m} \int_{t^{n-1}}^{t^{n}}\left\|u-U_{h}^{n}\right\|_{\Omega}^{2} d t \\
\leq & \left\|u_{0}-U_{h}^{0}\right\|_{L^{2}(\Omega)}^{2}+\sum_{n=1}^{m} \tau_{n} \eta_{\text {time }}^{n}+C \sum_{n=1}^{m} \tau_{n} \eta_{\text {space }}^{n} \\
+ & 2\left(\sum_{n=1}^{m} \int_{t^{n-1}}^{t^{n}}\left\|f-\bar{f}^{n}\right\|_{L^{2}(\Omega)} d t\right)^{2}
\end{aligned}
$$

holds, where the time error indicator $\eta_{\mathrm{time}}^{n}$ and space error indicator $\eta_{\mathrm{space}}^{n}$ are given by

$$
\eta_{\mathrm{time}}^{n}=\frac{1}{3}\left\|U_{h}^{n}-U_{h}^{n-1}\right\|_{\Omega}^{2}, \quad \eta_{\mathrm{space}}^{n}=\sum_{e \in \mathcal{B}^{n}} \eta_{e}^{n}
$$

with the local error indicator $\eta_{e}^{n}$ defined as

$$
\eta_{e}^{n}=\frac{1}{2} \sum_{K \in \Omega_{e}} h_{K}^{2}\left\|R^{n}\right\|_{L^{2}(K)}^{2}+h_{e}\left\|J_{e}^{n}\right\|_{L^{2}(e)}^{2} .
$$

Here $\Omega_{e}$ is the collection of two elements sharing the common side $e \in \mathcal{B}^{n}$.

Proof. For the sake of completeness, we sketch the proof here. From (2.2) we know that, for any $\varphi \in H_{0}^{1}(\Omega)$ and $v \in V_{0}^{n}$,

$$
\left\langle\frac{U_{h}^{n}-U_{h}^{n-1}}{\tau_{n}}, \varphi\right\rangle+\left\langle a \nabla U_{h}^{n}, \nabla \varphi\right\rangle=-\left\langle R^{n}, \varphi-v\right\rangle+\left\langle a \nabla U_{h}^{n}, \nabla(\varphi-v)\right\rangle+\left\langle\bar{f}^{n}, \varphi\right\rangle .
$$

For any $t \in\left(t^{n-1}, t^{n}\right]$, we denote by

$$
U_{h}(t)=l(t) U_{h}^{n}+(1-l(t)) U_{h}^{n-1}, \quad l(t)=\left(t-t^{n-1}\right) / \tau_{n} .
$$

Then from (2.1) and (2.5) we have, for a.e. $t \in\left(t^{n-1}, t^{n}\right]$, and for any $\varphi \in H_{0}^{1}(\Omega), v \in$ $V_{0}^{n}$,

$$
\left\langle\frac{\partial\left(u-U_{h}\right)}{\partial t}, \varphi\right\rangle+\left\langle a \nabla\left(u-U_{h}^{n}\right), \nabla \varphi\right\rangle=\left\langle R^{n}, \varphi-v\right\rangle-\left\langle a \nabla U_{h}^{n}, \nabla(\varphi-v)\right\rangle+\left\langle f-\bar{f}^{n}, \varphi\right\rangle .
$$

Now we resort to the Clément interpolant $\Pi^{n}: H_{0}^{1}(\Omega) \rightarrow V_{0}^{n}$, which satisfies the following local approximation properties, for any $\varphi \in H_{0}^{1}(\Omega)$ :

$$
\begin{aligned}
& \left\|\varphi-\Pi^{n} \varphi\right\|_{L^{2}(K)}+h_{K}\left\|\nabla\left(\varphi-\Pi^{n} \varphi\right)\right\|_{L^{2}(K)} \leq C^{*} h_{K}\|\nabla \varphi\|_{L^{2}(N(K))}, \\
& \left\|\varphi-\Pi^{n} \varphi\right\|_{L^{2}(e)} \leq C^{*} h_{e}^{1 / 2}\|\nabla \varphi\|_{L^{2}(N(e))},
\end{aligned}
$$

where $N(A)$ is the union of all elements in $\mathcal{M}^{n}$ surrounding the sets $A=K \in \mathcal{M}^{n}$ or $A=e \in \mathcal{B}^{n}$ (see Clément [10]). The constant $C^{*}$ depends only on the minimum angle of mesh $\mathcal{M}^{n}$. Based on this interpolant, by taking $\varphi=u-U_{h} \in H_{0}^{1}(\Omega)$, $v=\Pi^{n}\left(u-U_{h}\right) \in V_{0}^{n}$, and using (2.3) and the identity

$$
\left\langle a \nabla\left(u-U_{h}^{n}\right), \nabla\left(u-U_{h}\right)\right\rangle=\frac{1}{2}\left\|u-U_{h}^{n}\right\|_{\Omega}^{2}+\frac{1}{2}\left\|u-U_{h}\right\|_{\Omega}^{2}-\frac{1}{2}\left\|U_{h}-U_{h}^{n}\right\|_{\Omega}^{2},
$$


we deduce that

$$
\begin{aligned}
& \frac{1}{2} \frac{d}{d t}\left\|u-U_{h}\right\|_{L^{2}(\Omega)}^{2}+\frac{1}{2}\left\|u-U_{h}^{n}\right\|_{\Omega}^{2}+\frac{1}{2}\left\|u-U_{h}\right\|_{\Omega}^{2} \\
= & \frac{1}{2}\left\|U_{h}-U_{h}^{n}\right\|_{\Omega}^{2}+\left\langle R^{n},\left(u-U_{h}\right)-\Pi^{n}\left(u-U_{h}\right)\right\rangle \\
+ & \sum_{e \in \mathcal{B}^{n}} \int_{e} J_{e}^{n}\left[\left(u-U_{h}\right)-\Pi^{n}\left(u-U_{h}\right)\right] d s+\left\langle f-\bar{f}^{n}, u-U_{h}\right\rangle .
\end{aligned}
$$

For any $t^{*} \in\left(t^{m-1}, t^{m}\right]$, by integrating (2.7) in time from 0 to $t^{*}$, using (2.5)-(2.6), and exploiting the standard argument in finite element a posteriori analysis, we have

$$
\begin{aligned}
& \frac{1}{2}\left\|(u-U)\left(t^{*}\right)\right\|_{L^{2}(\Omega)}^{2}+\frac{1}{2} \sum_{n=1}^{m} \int_{t^{n-1}}^{t^{n} \wedge t^{*}}\left(\left\|u-U_{h}^{n}\right\|_{\Omega}^{2}+\left\|u-U_{h}\right\|_{\Omega}^{2}\right) d t \\
\leq & \frac{1}{2}\left\|u_{0}-U_{h}^{0}\right\|_{L^{2}(\Omega)}^{2}+\frac{1}{2} \sum_{n=1}^{m} \int_{t^{n-1}}^{t^{n}}\left\|U_{h}-U_{h}^{n}\right\|_{\Omega}^{2} d t \\
+ & C \sum_{n=1}^{m} \int_{t^{n-1}}^{t^{n}}\left(\eta_{\mathrm{space}}^{n}\right)^{1 / 2}\left\|u-U_{h}\right\|_{\Omega} d t+\frac{1}{4} \max _{0 \leq t \leq t^{*}}\left\|u-U_{h}\right\|_{L^{2}(\Omega)}^{2} \\
+ & \left(\sum_{n=1}^{m} \int_{t^{n-1}}^{t^{n}}\left\|f-\bar{f}^{n}\right\|_{L^{2}(\Omega)} d t\right)^{2},
\end{aligned}
$$

where $t^{n} \wedge t^{*}=\min \left(t^{n}, t^{*}\right)$. This implies the desired estimate (2.4) by using the fact that

$$
\int_{t^{n-1}}^{t^{n}}\left\|U_{h}-U_{h}^{n}\right\|_{\Omega}^{2} d t=\int_{t^{n-1}}^{t^{n}}(1-l(t))^{2}\left\|U_{h}^{n}-U_{h}^{n-1}\right\|_{\Omega}^{2} d t=\frac{1}{3} \tau_{n}\left\|U_{h}^{n}-U_{h}^{n-1}\right\|_{\Omega}^{2} .
$$

This completes the proof.

To conclude this section we give a few remarks about the a posteriori error estimates derived.

Remark 2.1. Let $P^{n}: L^{2}(\Omega) \rightarrow V_{0}^{n}$ be the $L^{2}$ projection operator. The coarsening errors involving $U_{h}^{n-1}-P^{n} U_{h}^{n-1}$ which appeared in previous studies [7, [12], [16] are not present in our a posteriori error estimates. They are hidden in the space error term $\left\|h_{n} R^{n}\right\|_{L^{2}(\Omega)}^{2}$ and the time error term $\left\|U_{h}^{n}-U_{h}^{n-1}\right\|_{\Omega}^{2}$. Here $h_{n}$ is the piecewise constant function which is equal to $h_{K}$ on each $K \in \mathcal{M}^{n}$.

Remark 2.2. The constant $C$ in the a posteriori error estimate (2.4) is proportional to the maximum jump of the coefficients $\Lambda=\max _{x \in \bar{\Omega}} a(x) / \min _{x \in \bar{\Omega}} a(x)$. This dependence is rather undesirable for strongly discontinuous coefficients and indeed can be removed by modifying the associated space error indicator (see Chen and Dai [6] for a study on a posteriori error analysis and adaptivity for elliptic problems with strongly discontinuous coefficients). In order to avoid unnecessary and inessential complications of the argument, we will not elaborate on this issue in this paper.

Remark 2.3. In our a posteriori error estimate at the $n$-th time step, the time discretization error is controlled by $\left\|U_{h}^{n}-U_{h}^{n-1}\right\|_{\Omega}$ and $\int_{t^{n-1}}^{t^{n}}\left\|f-\bar{f}^{n}\right\|_{L^{2}(\Omega)} d t$, which can only be reduced by reducing the time-step sizes $\tau_{n}$. On the other hand, the time-step size $\tau_{n}$ essentially controls the semidiscretization error: the error between the exact solution $u$ and the solution $U^{n}$ of (1.3). Thus $\left\|U_{h}^{n}-U_{h}^{n-1}\right\|_{\Omega}$ is not a 
good error indicator for time discretization unless the space-discretization error is sufficiently resolved. In the adaptive method for time-dependent problems, we must do space mesh and time-step size adaptation simultaneously. Ignoring either one of them may not provide correct error control of approximation to the problem.

Remark 2.4. For given $U_{h}^{n-1} \in V_{0}^{n-1}$, let $U_{*}^{n} \in H_{0}^{1}(\Omega)$ be the solution of the continuous problem (1.4). Then the space error indicator $\eta_{\text {space }}^{n}$ controls only the error between $U_{h}^{n}$ and $U_{*}^{n}$, not between $U_{h}^{n}$ and $U^{n}$ (or the exact solution $u$ ). This observation plays an important role in the subsequent analysis.

2.2. A lower bound (space error). The objective of this subsection is to prove the following estimate for the local error which ensures over-refinement will not occur for the refinement strategy based on our space error indicator. For any $K \in \mathcal{M}^{n}$ and $\varphi \in L^{2}(\Omega)$, we define $P_{K} \varphi=\frac{1}{|K|} \int_{K} \varphi d x$, the average of $\varphi$ over $K$. For any $n=1,2, \ldots$, we also need the notation

$$
\hat{C}_{n}=\max _{K \in \mathcal{M}^{n}}\left(h_{K}^{2} / \tau_{n}\right), \quad h_{K}=\operatorname{diam}(K) .
$$

Theorem 2.2. There exist constants $C_{2}, C_{3}>0$ depending only on the minimum angle of $\mathcal{M}^{n}$ and the coefficient $a(x)$ such that for any $e \in \mathcal{B}^{n}$, the following estimate holds:

$$
\begin{aligned}
\eta_{e}^{n} \leq & C_{2} \hat{C}_{n} \sum_{K \in \Omega_{e}}\left(\frac{1}{\tau_{n}}\left\|U_{*}^{n}-U_{h}^{n}\right\|_{L^{2}(K)}^{2}+\left\|U_{*}^{n}-U_{h}^{n}\right\|_{K}^{2}\right) \\
& +C_{3} \sum_{K \in \Omega_{e}} h_{K}^{2}\left\|R^{n}-P_{K} R^{n}\right\|_{L^{2}(K)}^{2} .
\end{aligned}
$$

Before we proceed to the proof, we remark that the dependence of $C_{2}, C_{3}$ on the coefficient in the case of strongly discontinuous coefficients can be traced by the techniques developed in [6] for elliptic problems. We also remark that our form of the lower bound (2.9) differs from the one in [17, where the space error indicator is bounded by the sum of the energy-error and the time-error indicator $\left\|U_{h}^{n}-U_{h}^{n-1}\right\|_{\Omega}$.

Proof. The proof makes use of the idea in Verfürth [19. For any $K \in \mathcal{M}^{n}$, let $\psi_{K}=(d+1)^{d+1} \lambda_{1} \cdots \lambda_{d+1}$ be the bubble function, where $\lambda_{1}, \ldots, \lambda_{d+1}$ are the barycentric coordinate functions. By the standard scaling argument, we have the following inf-sup relation that holds for some constant $\beta$ depending only on the minimum angle of $K \in \mathcal{M}^{n}$ :

$$
\inf _{v_{h} \in P_{1}(K)} \sup _{\varphi_{h} \in P_{1}(K)} \frac{\int_{K} v_{h} \varphi_{h} \psi_{K} d x}{\left\|\varphi_{h}\right\|_{L^{2}(K)}\left\|v_{h}\right\|_{L^{2}(K)}} \geq \beta>0 .
$$

Thus there exists a function $\varphi^{n} \in P_{1}(K)$ with $\left\|\varphi^{n}\right\|_{L^{2}(K)}=1$ such that

$$
\begin{aligned}
& \beta\left\|P_{K} R^{n}\right\|_{L^{2}(K)} \\
\leq & \int_{K}\left(P_{K} R^{n}\right) \psi_{K} \varphi^{n} d x \\
= & \int_{K}\left(P_{K} R^{n}-R^{n}\right) \psi_{K} \varphi^{n} d x+\int_{K}\left(\bar{f}^{n}-\frac{U_{h}^{n}-U_{h}^{n-1}}{\tau_{n}}\right) \psi_{K} \varphi^{n} d x \\
= & \int_{K}\left(P_{K} R^{n}-R^{n}\right) \psi_{K} \varphi^{n} d x+\int_{K} \frac{U_{*}^{n}-U_{h}^{n}}{\tau_{n}} \psi_{K} \varphi^{n} d x+\left\langle a \nabla U_{*}^{n}, \nabla\left(\psi_{K} \varphi^{n}\right)\right\rangle_{K},
\end{aligned}
$$


where we have used (1.4) in the last identity. Since $U_{h}^{n} \in P_{1}(K)$ and $\psi_{K}=0$ on $\partial K$, simple integration by parts implies that $\left\langle a \nabla U_{h}^{n}, \nabla\left(\psi_{K} \varphi^{n}\right)\right\rangle_{K}=0$. Thus, we have

$$
\begin{aligned}
\left\|P_{K} R^{n}\right\|_{L^{2}(K)} \leq & C\left\|R^{n}-P_{K} R^{n}\right\|_{L^{2}(K)}+C \tau_{n}^{-1}\left\|U_{*}^{n}-U_{h}^{n}\right\|_{L^{2}(K)} \\
& +C\left\|U_{*}^{n}-U_{h}^{n}\right\|_{K}\left\|\psi_{K} \varphi^{n}\right\|_{K} .
\end{aligned}
$$

Since $\left\|\psi_{K} \varphi^{n}\right\|_{K} \leq C h_{K}^{-1}$ by inverse estimate, we conclude by the definition of $\hat{C}_{n}$ in (2.8) that

$$
\begin{aligned}
\left\|P_{K} R^{n}\right\|_{L^{2}(K)} \leq & C\left\|R^{n}-P_{K} R^{n}\right\|_{L^{2}(K)} \\
& +C \hat{C}_{n}^{1 / 2} h_{K}^{-1}\left(\frac{1}{\tau_{n}}\left\|U_{*}^{n}-U_{h}^{n}\right\|_{L^{2}(K)}^{2}+\left\|U_{*}^{n}-U_{h}^{n}\right\|_{K}^{2}\right)^{1 / 2} .
\end{aligned}
$$

Therefore, we have

$$
\begin{aligned}
h_{K}^{2}\left\|R^{n}\right\|_{L^{2}(K)}^{2} \leq & C h_{K}^{2}\left\|R^{n}-P_{K} R^{n}\right\|_{L^{2}(K)}^{2} \\
& +C \hat{C}_{n}\left(\frac{1}{\tau_{n}}\left\|U_{*}^{n}-U_{h}^{n}\right\|_{L^{2}(K)}^{2}+\left\|U_{*}^{n}-U_{h}^{n}\right\|_{K}^{2}\right) .
\end{aligned}
$$

For any $e \in \mathcal{B}^{n}$, let $\psi_{e}=d^{d} \lambda_{1} \cdots \lambda_{d}$ be the bubble function, where $\lambda_{1}, \ldots, \lambda_{d}$ are the barycentric coordinate functions associated with the nodes of $e$. Denote by $\psi^{n}=J_{e}^{n} \psi_{e} \in H_{0}^{1}(\Omega)$. Then, since $J_{e}^{n}$ is constant on $e \in \mathcal{B}^{n}$, we get, after integration by parts, that

$$
\begin{aligned}
\left\|J_{e}^{n}\right\|_{L^{2}(e)}^{2} & \leq C \int_{e} J_{e}^{n} \psi^{n} d x=-C \sum_{K \in \Omega_{e}} \int_{K} a(x) \nabla U_{h}^{n} \nabla \psi^{n} d x \\
& =C \sum_{K \in \Omega_{e}} \int_{K} a(x) \nabla\left(U_{*}^{n}-U_{h}^{n}\right) \nabla \psi^{n} d x-C \sum_{K \in \Omega_{e}} \int_{K} R^{n} \psi^{n} d x,
\end{aligned}
$$

where we have used the definition of $U_{*}^{n}$ in (1.4). Moreover, it is easy to see that $\left\|\nabla \psi^{n}\right\|_{L^{2}(K)} \leq C h_{e}^{-1 / 2}\left\|J_{e}^{n}\right\|_{L^{2}(e)}, \quad\left\|\psi^{n}\right\|_{L^{2}(K)} \leq C h_{e}^{1 / 2}\left\|J_{e}^{n}\right\|_{L^{2}(e)} \forall K \in \Omega_{e}$.

Thus

$$
h_{e}\left\|J_{e}^{n}\right\|_{L^{2}(e)}^{2} \leq C \sum_{K \in \Omega_{e}}\left(h_{K}^{2}\left\|R^{n}\right\|_{L^{2}(K)}^{2}+\left\|U_{*}^{n}-U_{h}^{n}\right\|_{K}^{2}\right) .
$$

This completes the proof.

Summing up (2.9) for all $e \in \mathcal{B}^{n}$, we obtain that the following important estimate

$$
\eta_{\text {space }}^{n} \leq 2 C_{2} \hat{C}_{n}\left\|U_{*}^{n}-U_{h}^{n}\right\|_{\tau_{n}, \Omega}^{2}+2 C_{3} \operatorname{osc}\left(R^{n}, \mathcal{M}^{n}\right)^{2},
$$

where the weighted norm $\left\|U_{*}^{n}-U_{h}^{n}\right\|_{\tau_{n}, \Omega}$ is defined in (1.7), and the oscillation of the residual $\operatorname{osc}\left(R^{n}, \mathcal{M}^{n}\right)$ is defined in (1.5).

\section{Adaptive Algorithm}

We start by considering the algorithm for time-step size control. The adjustment of the time-step size is based on the error equidistribution strategy: the time discretization error should be equally distributed to each time interval 
$\left(t^{n-1}, t^{n}\right), n=1, \ldots, N$. Let $\mathrm{TOL}_{\text {time }}$ be the total tolerance allowed for the part of a posteriori error estimate in (2.4) related to the time discretization; that is,

$$
\sum_{n=1}^{N} \tau_{n} \eta_{\text {time }}^{n}+2\left(\sum_{n=1}^{N} \int_{t^{n-1}}^{t^{n}}\left\|f-\bar{f}^{n}\right\|_{L^{2}(\Omega)} d t\right)^{2} \leq \mathrm{TOL}_{\text {time }} .
$$

A natural way to achieve (3.1) is to adjust the time-step size $\tau_{n}$ such that the following relations are satisfied:

$$
\eta_{\text {time }}^{n} \leq \frac{\text { TOL }_{\text {time }}}{2 T}, \quad \frac{1}{\tau_{n}} \int_{t^{n-1}}^{t^{n}}\left\|f-\bar{f}^{n}\right\|_{L^{2}(\Omega)} d t \leq \frac{1}{2 T} \sqrt{\text { TOL }_{\text {time }}} .
$$

The following algorithm can be used to control the time-step size at each time step $n$ (cf., e.g., [18).

Algorithm 3.1 (Time-step size control). Prescribe tolerance TOL $\mathrm{L}_{\text {time }}$, parameters $\delta_{1} \in(0,1), \delta_{2}>1$, and $\theta_{\text {time }} \in(0,1)$.

$\tau_{n}:=\tau_{n-1}$

solve the time discretization problem and estimate the error

while (3.2) is not satisfied do

$\tau_{n}:=\delta_{1} \tau_{n}$

solve the time discretization problem and estimate the error

end while

if the relations

$\eta_{\text {time }}^{n} \leq \theta_{\text {time }} \frac{\text { TOL }_{\text {time }}}{2 T}, \frac{1}{\tau_{n}} \int_{t^{n-1}}^{t^{n}}\left\|f-\bar{f}^{n}\right\|_{L^{2}(\Omega)} d t \leq \frac{1}{2 T} \sqrt{\theta_{\text {time }} \text { TOL }_{\text {time }}}$

are satisfied then

$$
\tau_{n}:=\delta_{2} \tau_{n}
$$

end if

A good choice of the parameters in the algorithm above for the backward Euler scheme in time is to take $\delta_{1}=0.5, \delta_{2}=2$, and $\theta_{\text {time }}=0.5$. Algorithm 3.1 controls only the time-step size, which must be combined with some algorithm for the mesh adaptation in practical applications. The main difficulty now is the mesh coarsening, which increases the error. If the mesh adaptation procedure iterates several times, it may occur that elements which were marked for coarsening in the beginning must be refined at the end to reduce the error, which is certainly undesirable. One possibility, which is proposed in [18], for overcoming this difficulty is to delay the mesh coarsening until the final iteration of the adaptive procedure, allowing refinements before only. Let $\mathrm{TOL}_{\text {space }}$ be the tolerance allowed for the part of the a posteriori error estimate in (2.4) related to the space discretization. Then the usual stopping criterion for the mesh adaptation is to satisfy the following relation at each time step $n$ :

$$
\eta_{\text {space }}^{n} \leq \frac{\mathrm{TOL}_{\text {space }}}{T} .
$$

This stopping rule is appropriate for mesh refinements but not for mesh coarsening. In this paper we will use a new coarsening error indicator based on the following theorem. 
Theorem 3.1. Given $U_{h}^{n-1} \in V_{0}^{n-1}$ and $\tau_{n}>0$, let $\mathcal{M}_{H}^{n}$ be a coarsening of the mesh $\mathcal{M}^{n}$. Let $U_{H}^{n} \in V_{0}^{n, H}, U_{h}^{n} \in V_{0}^{n}$ be the solutions of the discrete problem (1.2) over meshes $\mathcal{M}_{H}^{n}$ and $\mathcal{M}^{n}$, respectively. Then the following error estimate is valid

$$
\left\|U_{*}^{n}-U_{H}^{n}\right\|_{\tau_{n}, \Omega}^{2} \leq\left\|U_{*}^{n}-U_{h}^{n}\right\|_{\tau_{n}, \Omega}^{2}+\left\|U_{h}^{n}-I_{H}^{n} U_{h}^{n}\right\|_{\tau_{n}, \Omega}^{2}
$$

where $I_{H}^{n}: C(\bar{\Omega}) \rightarrow V^{n, H}$ is the standard linear finite element interpolant, and the weighted norm $\|\cdot\|_{\tau_{n}, \Omega}$ is defined in (1.7).

Proof. By definition, $U_{H}^{n} \in V_{0}^{n, H}$ and $U_{h}^{n} \in V_{0}^{n}$ satisfy the relations

$$
\begin{array}{ll}
\left\langle\frac{U_{H}^{n}-U_{h}^{n-1}}{\tau_{n}}, v\right\rangle+\left\langle a \nabla U_{H}^{n}, \nabla v\right\rangle=\left\langle\bar{f}^{n}, v\right\rangle & \forall v \in V_{0}^{n, H}, \\
\left\langle\frac{U_{h}^{n}-U_{h}^{n-1}}{\tau_{n}}, v\right\rangle+\left\langle a \nabla U_{h}^{n}, \nabla v\right\rangle=\left\langle\bar{f}^{n}, v\right\rangle & \forall v \in V_{0}^{n},
\end{array}
$$

Since $\mathcal{M}_{H}^{n}$ is a coarsening of $\mathcal{M}^{n}$, we have $V_{0}^{n, H} \subset V_{0}^{n}$. Thus $U_{H}^{n}-U_{h}^{n} \in V_{0}^{n}$. Now the equation (3.6) together with (1.4) implies the Galerkin orthogonal identity

$$
\left\langle\frac{U_{*}^{n}-U_{h}^{n}}{\tau_{n}}, U_{H}^{n}-U_{h}^{n}\right\rangle+\left\langle a \nabla\left(U_{*}^{n}-U_{h}^{n}\right), \nabla\left(U_{H}^{n}-U_{h}^{n}\right)\right\rangle=0 .
$$

Hence

$$
\left\|U_{*}^{n}-U_{H}^{n}\right\|_{\tau_{n}, \Omega}^{2}=\left\|U_{*}^{n}-U_{h}^{n}\right\|_{\tau_{n}, \Omega}^{2}+\left\|U_{H}^{n}-U_{h}^{n}\right\|_{\tau_{n}, \Omega}^{2}
$$

Next, by subtracting (3.5) from (3.6) and taking $v=U_{H}^{n}-I_{H}^{n} U_{h}^{n} \in V_{0}^{n, H}$, we obtain the Galerkin orthogonal relation

$$
\left\langle\frac{U_{H}^{n}-U_{h}^{n}}{\tau_{n}}, U_{H}^{n}-I_{H}^{n} U_{h}^{n}\right\rangle+\left\langle a \nabla\left(U_{H}^{n}-U_{h}^{n}\right), \nabla\left(U_{H}^{n}-I_{H}^{n} U_{h}^{n}\right)\right\rangle=0,
$$

which implies

$$
\begin{aligned}
\left\|U_{H}^{n}-U_{h}^{n}\right\|_{\tau_{n}, \Omega}^{2} & =\left\|U_{h}^{n}-I_{H}^{n} U_{h}^{n}\right\|_{\tau_{n}, \Omega}^{2}-\left\|U_{H}^{n}-I_{H}^{n} U_{h}^{n}\right\|_{\tau_{n}, \Omega}^{2} \\
& \leq\left\|U_{h}^{n}-I_{H}^{n} U_{h}^{n}\right\|_{\tau_{n}, \Omega}^{2} .
\end{aligned}
$$

This completes the proof by using (3.7).

Theorem 3.1 suggests that we introduce the coarsening error indicator

$$
\eta_{\text {coarse }}^{n}=\frac{1}{\tau_{n}}\left\|I_{H}^{n} U_{h}^{n}-U_{h}^{n}\right\|_{L^{2}(\Omega)}^{2}+\left\|I_{H}^{n} U_{h}^{n}-U_{h}^{n}\right\|_{\Omega}^{2} .
$$

The nice feature of this indicator is that it does not depend on $U_{H}^{n}$, the solution of the coarsened problem. This property allows us to coarsen only once, without checking whether the coarsened solution $U_{H}^{n}$ satisfies some stopping criterion such as (3.4). Combining the ideas above, we arrive at the following adaptive algorithm for one single time step, which modifies the procedure proposed in [18].

Algorithm 3.2 (Time and space adaptive algorithm). Prescribe tolerances TOL $\mathrm{L}_{\mathrm{time}}$, TOL $_{\text {space }}$ and TOL $\mathrm{L}_{\text {coarse }}$, parameters $\delta_{1} \in(0,1), \delta_{2}>1$ and $\theta_{\text {time }} \in(0,1)$. Let $U_{h}^{n-1}$ be computed from the previous time step at time $t^{n-1}$ with the mesh $\mathcal{M}^{n-1}$ and the time-step size $\tau_{n-1}$.

1. $\mathcal{M}^{n}:=\mathcal{M}^{n-1}, \tau_{n}:=\tau_{n-1}, t^{n}:=t^{n-1}+\tau_{n}$ solve the discrete problem for $U_{h}^{n}$ on $\mathcal{M}^{n}$ using data $U_{h}^{n-1}$ compute error estimates on $\mathcal{M}^{n}$ 
2. while (3.2) is not satisfied do

$\tau_{n}:=\delta_{1} \tau_{n}, t^{n}:=t^{n-1}+\tau_{n}$

solve the discrete problem for $U_{h}^{n}$ on $\mathcal{M}^{n}$ using data $U_{h}^{n-1}$

compute error estimates on $\mathcal{M}^{n}$

end while

3. while $\eta_{\text {space }}^{n}>\mathrm{TOL}_{\text {space }} / T$ do

refine mesh $\mathcal{M}^{n}$ producing a modified $\mathcal{M}^{n}$

solve the discrete problem for $U_{h}^{n}$ on $\mathcal{M}^{n}$ using data $U_{h}^{n-1}$

compute error estimates on $\mathcal{M}^{n}$

while 3.2 is not satisfied do

$\tau_{n}:=\delta_{1} \tau_{n}, t^{n}:=t^{n-1}+\tau_{n}$

solve the discrete problem for $U_{h}^{n}$ on $\mathcal{M}^{n}$ using data $U_{h}^{n-1}$

compute error estimates on $\mathcal{M}^{n}$

end while

end while

4. coarsen $\mathcal{M}^{n}$ producing a modified mesh $\mathcal{M}^{n}$ according to $\eta_{\text {coarse }}^{n} \leq \frac{\mathrm{TOL}_{\text {coarse }}}{T}$ solve the discrete problem for $U_{h}^{n}$ on $\mathcal{M}^{n}$ using data $U_{h}^{n-1}$

5. if 3.3 is satisfied then

end if

$$
\tau_{n}:=\delta_{2} \tau_{n}
$$

The goal of the first three steps in the algorithm above is to reduce the timestep size and refine the mesh so that the time and space error indicators become smaller than the respective tolerances. We achieve this goal by first reducing the time-step size to have the time error estimate below the tolerance while keeping the mesh unchanged. In Step 5, when the time error indicator is much smaller than the tolerance, the step size is enlarged (coarsened) by a factor $\delta_{2}>1$. In this case, the actual time step is not recalculated; only the initial time-step size for the next time step is changed.

We have the following theorem which guarantees the reliability of the algorithm above in terms of error control.

Theorem 3.2. For $n \geq 1$, assume Algorithm 3.2 terminates and generates the final mesh $\mathcal{M}_{H}^{n}$, time-step size $\tau_{n}$, and the the corresponding discrete solution $U_{H}^{n}$. The mesh $\mathcal{M}_{H}^{n}$ is coarsened from the mesh $\mathcal{M}^{n}$ produced by the first three steps. Then for any integer $1 \leq m \leq N$, there exists a constant $C$ depending only on the minimum angles of $\mathcal{M}^{n}, n=1,2, \ldots, m$, and the coefficient $a(x)$ such that the estimate

$$
\begin{aligned}
& \frac{1}{2}\left\|u^{m}-U_{H}^{m}\right\|_{L^{2}(\Omega)}^{2}+\sum_{n=1}^{m} \int_{t^{n-1}}^{t^{n}}\left\|u-U_{H}^{n}\right\|_{\Omega}^{2} d t \\
& \quad \leq\left\|u_{0}-U_{h}^{0}\right\|_{L^{2}(\Omega)}^{2}+\frac{t^{m}}{T} \mathrm{TOL}_{\text {time }}+C \frac{t^{m}}{T} \mathrm{TOL}_{\text {space }}+C \hat{C}_{H}^{m} \frac{t^{m}}{T} \mathrm{TOL}_{\text {coarse }}
\end{aligned}
$$

holds, where $\hat{C}_{H}^{m}=\max \left\{h_{K}^{2} / \tau_{n}: K \in \mathcal{M}_{H}^{n}, n=1,2, \ldots, m\right\}$.

Proof. Let $U_{h}^{n}$ be the solution of the discrete problem (2.2) over the mesh $\mathcal{M}^{n}$ and with the time-step size $\tau_{n}$. Then upon the termination of Algorithm 3.2 we have that

$\eta_{\text {time }}^{n} \leq \frac{\text { TOL }_{\text {time }}}{2 T}, \frac{1}{\tau_{n}} \int_{t^{n-1}}^{t^{n}}\left\|f-\bar{f}^{n}\right\|_{L^{2}(\Omega)} d t \leq \frac{1}{2 T} \sqrt{\text { TOL }_{\text {time }}}, \quad \eta_{\text {space }}^{n} \leq \frac{\text { TOL }_{\text {space }}}{T}$. 
From (2.2) we know that for any $\varphi \in H_{0}^{1}(\Omega)$

$$
\begin{aligned}
& \left\langle\frac{U_{H}^{n}-U_{h}^{n-1}}{\tau_{n}}, \varphi\right\rangle+\left\langle a \nabla U_{H}^{n}, \nabla \varphi\right\rangle \\
= & \left\langle\frac{U_{H}^{n}-U_{h}^{n}}{\tau_{n}}, \varphi\right\rangle+\left\langle a \nabla\left(U_{H}^{n}-U_{h}^{n}\right), \nabla \varphi\right\rangle \\
- & \left\langle R^{n}, \varphi\right\rangle+\left\langle a \nabla U_{h}^{n}, \nabla \varphi\right\rangle+\left\langle\bar{f}^{n}, \varphi\right\rangle .
\end{aligned}
$$

Since $\mathcal{M}_{H}^{n}$ is a coarsening of $\mathcal{M}^{n}$, by the Galerkin orthogonal relation, as in Theorem 3.1, we have

$$
\left\langle\frac{U_{H}^{n}-U_{h}^{n}}{\tau_{n}}, v_{H}\right\rangle+\left\langle a \nabla\left(U_{H}^{n}-U_{h}^{n}\right), \nabla v_{H}\right\rangle=0 \quad \forall v_{H} \in V_{0}^{n, H} .
$$

On the other hand, since $U_{h}^{n}$ is the discrete solution over mesh $\mathcal{M}^{n}$, we have

$$
-\left\langle R^{n}, v\right\rangle+\left\langle a \nabla U_{h}^{n}, \nabla v\right\rangle=0 \quad \forall v \in V_{0}^{n} .
$$

Thus from (2.1) and (3.10) we deduce that for a.e. $t \in\left(t^{n-1}, t^{n}\right]$ and for any $\varphi \in H_{0}^{1}(\Omega), v_{H} \in V_{0}^{n, H}, v \in V_{0}^{n}$,

$$
\begin{aligned}
& \left\langle\frac{\partial\left(u-U_{H}\right)}{\partial t}, \varphi\right\rangle+\left\langle a \nabla\left(u-U_{H}^{n}\right), \nabla \varphi\right\rangle \\
= & \left\langle R^{n}, \varphi-v\right\rangle-\left\langle a \nabla U_{h}^{n}, \nabla(\varphi-v)\right\rangle+\left\langle f-\bar{f}^{n}, \varphi\right\rangle \\
- & \left\langle\frac{U_{H}^{n}-U_{h}^{n}}{\tau_{n}}, \varphi-v_{H}\right\rangle-\left\langle a \nabla\left(U_{H}^{n}-U_{h}^{n}\right), \nabla\left(\varphi-v_{H}\right)\right\rangle,
\end{aligned}
$$

where for any $t \in\left(t^{n-1}, t^{n}\right], U_{H}(t)=l(t) U_{H}^{n}+(1-l(t)) U_{h}^{n-1}$ with $l(t)=$ $\left(t-t^{n-1}\right) / \tau_{n}$. By taking $v_{H}=\Pi_{H}^{n} \varphi \in V_{0}^{n, H}$, the Clément interpolation of $\varphi \in H_{0}^{1}(\Omega)$ in $V_{0}^{n, H}$, we get, after using the estimate (2.5) for the Clément interpolant, that

$$
\begin{aligned}
& \left|\left\langle\frac{U_{H}^{n}-U_{h}^{n}}{\tau_{n}}, \varphi-\Pi_{H}^{n} \varphi\right\rangle+\left\langle a \nabla\left(U_{H}^{n}-U_{h}^{n}\right), \nabla\left(\varphi-\Pi_{H}^{n} \varphi\right)\right\rangle\right| \\
\leq & C\left(\sum_{K \in \mathcal{M}_{H}^{n}} h_{K}^{2} \tau_{n}^{-2}\left\|U_{H}^{n}-U_{h}^{n}\right\|_{L^{2}(K)}^{2}+\left\|U_{H}^{n}-U_{h}^{n}\right\|_{\Omega}^{2}\right)^{1 / 2}\|\varphi\|_{\Omega} \\
\leq & C\left(\hat{C}_{H}^{m}\right)^{1 / 2}\left\|U_{H}^{n}-U_{h}^{n}\right\|_{\tau_{n}, \Omega}\|\varphi\|_{\Omega} .
\end{aligned}
$$

Again, since $\mathcal{M}_{H}^{n}$ is a coarsening of $\mathcal{M}^{n}$, from the proof of Theorem 3.1 and Step 4 in Algorithm 3.2 we know that

$$
\left\|U_{H}^{n}-U_{h}^{n}\right\|_{\tau_{n}, \Omega} \leq\left\|I_{H}^{n} U_{h}^{n}-U_{h}^{n}\right\|_{\tau_{n}, \Omega} \leq\left(\eta_{\text {coarse }}^{n}\right)^{1 / 2} \leq \sqrt{\frac{\text { TOL }_{\text {coarse }}}{T}},
$$

which yields

$$
\left|\left\langle\frac{U_{H}^{n}-U_{h}^{n}}{\tau_{n}}, \varphi-\Pi_{H}^{n} \varphi\right\rangle+\left\langle a \nabla\left(U_{H}^{n}-U_{h}^{n}\right), \nabla\left(\varphi-\Pi_{H}^{n} \varphi\right)\right\rangle\right| \leq C \sqrt{\frac{\hat{C}_{H}^{m} \mathrm{TOL}_{\text {coarse }}}{T}}\|\varphi\|_{\Omega} .
$$

The rest of the proof is similar to that of Theorem 2.1. Here we omit the details.

In practical computations, it is natural to choose the coarsening tolerance $\mathrm{TOL}_{\text {coarse }}$ much smaller than the space tolerance $\mathrm{TOL}_{\text {space }}$. However, the additional factor $\hat{C}_{H}^{m}$ in estimate (3.9) suggests that the ratio between the coarsening tolerance and the time tolerance should also be small; see Remark 4.7 


\section{Termination OF THE ADAPtive Algorithm}

The goal of this section is to show that for any given tolerances $\mathrm{TOL}_{\text {time }}, \mathrm{TOL}_{\text {space }}$ and $\mathrm{TOL}_{\text {coarse }}$, Algorithm 3.2 will terminate in a finite number of steps. This is a non-standard result in finite element theory for parabolic problems as it does not require the underlying finite element mesh size and the time-step size tends to zero. Since the algorithm only coarsens the mesh once in Step 4, we need only consider the termination of the refinements in the first three steps.

Let $\tau_{n, k}$ and $\mathcal{M}^{n, k}$ be the time-step size and mesh at the $k$-th iteration produced by the first three steps in the algorithm. The associated finite element space and discrete solution are denoted by $V^{n, k}$ and $U_{h}^{n, k}$, respectively. Let $t^{n, k}=t^{n-1}+\tau_{n, k}$. Throughout this section we will use the notation that $\phi^{n, k}=\phi\left(x, t^{n, k}\right)$ if $\phi$ is continuous in $\left(t^{n-1}, t^{n}\right]$ and $\bar{\phi}^{n, k}=\frac{1}{\tau_{n, k}} \int_{t^{n-1}}^{t^{n, k}} \phi(x, t) d t$ if $\phi \in L^{2}\left(t^{n-1}, t^{n, k} ; L^{2}(\Omega)\right)$.

By (1.2) we know that $U_{h}^{n, k} \in V_{0}^{n, k}=V^{n, k} \cap H_{0}^{1}(\Omega)$ satisfies

$$
\left\langle\frac{U_{h}^{n, k}-U_{h}^{n-1}}{\tau_{n, k}}, v\right\rangle+\left\langle a \nabla U_{h}^{n, k}, \nabla v\right\rangle=\left\langle\bar{f}^{n, k}, v\right\rangle \quad \forall v \in V_{0}^{n, k} .
$$

From Algorithm 3.2 we know that $\mathcal{M}^{n, 0}=\mathcal{M}^{n-1}, \tau_{n, 0}=\tau_{n-1}$ and

$$
\mathcal{M}^{n, k} \text { is a refinement of } \mathcal{M}^{n, k-1}, \quad \tau_{n, k}=\delta_{1} \tau_{n, k-1} \text { or } \tau_{n, k}=\tau_{n, k-1} \quad \forall k \geq 1 \text {. }
$$

4.1. Termination of time-step size refinements. Our first objective is to show that the iteration to find the time-step size in Algorithm 3.2 can be terminated in a finite number of steps. To begin with, we recall the following result.

Lemma 4.1. Assume that $f \in L^{2}\left(0, T ; L^{2}(\Omega)\right)$ and $w_{0} \in H_{0}^{1}(\Omega)$. Let $w$ be the solution of the linear parabolic problem

$$
\begin{array}{ll}
\frac{\partial w}{\partial t}-\operatorname{div}(a(x) \nabla w)=f & \text { in } \Omega \times(0, T), \\
w=0 \quad \text { on } \Gamma \times(0, T), & w(x, 0)=w_{0}(x) \quad \text { in } \Omega .
\end{array}
$$

Let $\bar{w}(x, t)=\frac{1}{t} \int_{0}^{t} w(x, s) d s$. Then we have

$$
\frac{1}{t}\left\|w(t)-w_{0}\right\|_{L^{2}(\Omega)}^{2}+\left\|\bar{w}(t)-w_{0}\right\|_{\Omega}^{2} \rightarrow 0 \quad \text { as } t \rightarrow 0 .
$$

Proof. Since $w_{0} \in H_{0}^{1}(\Omega)$, it is well known that $\partial_{t} w \in L^{2}\left(0, T ; L^{2}(\Omega)\right)$ and $w \in$ $L^{\infty}\left(0, T ; H_{0}^{1}(\Omega)\right)$. Thus

$$
\begin{aligned}
& \frac{1}{t}\left\|\bar{w}(t)-w_{0}\right\|_{L^{2}(\Omega)}^{2}+\frac{1}{t}\left\|w(t)-w_{0}\right\|_{L^{2}(\Omega)}^{2} \\
\leq & 2 \int_{0}^{t}\left\|\partial_{t} w\right\|_{L^{2}(\Omega)}^{2} d s \rightarrow 0 \quad \text { as } t \rightarrow 0 .
\end{aligned}
$$

Multiplying (4.2) by $\partial_{t} w$ and integrating over $\Omega \times(0, t)$, we get

$$
\int_{0}^{t}\left\|\frac{\partial w}{\partial t}\right\|_{L^{2}(\Omega)}^{2} d t+\frac{1}{2}\|w(t)\|_{\Omega}^{2}-\frac{1}{2}\left\|w_{0}\right\|_{\Omega}^{2}=\int_{0}^{t}\left\langle f, \frac{\partial w}{\partial t}\right\rangle d s .
$$

By letting $t \rightarrow 0$ in the equality above, we know that $\|w(t)\|_{\Omega} \rightarrow\left\|w_{0}\right\|_{\Omega}$ and thus $\|\bar{w}(t)\|_{\Omega} \rightarrow\left\|w_{0}\right\|_{\Omega}$ as $t \rightarrow 0$. Now we integrate (4.2) in time from 0 to $t$ to obtain

$$
\left\langle\frac{w(t)-w_{0}}{t}, \varphi\right\rangle+\langle a \nabla \bar{w}(t), \nabla \varphi\rangle=\langle\bar{f}(t), \varphi\rangle \quad \forall \varphi \in H_{0}^{1}(\Omega) .
$$


By (4.3), we have

$$
\frac{1}{t}\left\langle w(t)-w_{0}, \bar{w}(t)-w_{0}\right\rangle \rightarrow 0 \quad \text { and } \quad\left\langle\bar{f}(t), \bar{w}(t)-w_{0}\right\rangle \rightarrow 0 \quad \text { as } t \rightarrow 0 .
$$

Thus, by taking $\varphi=\bar{w}-w_{0}$ in (4.4) and letting $t \rightarrow 0$, we get

$$
\lim _{t \rightarrow 0}\left(\frac{1}{2}\|\bar{w}(t)\|_{\Omega}^{2}-\frac{1}{2}\left\|w_{0}\right\|_{\Omega}^{2}+\frac{1}{2}\left\|\bar{w}(t)-w_{0}\right\|_{\Omega}^{2}\right)=0,
$$

which yields $\left\|\bar{w}(t)-w_{0}\right\|_{\Omega} \rightarrow 0$ since $\|\bar{w}(t)\|_{\Omega} \rightarrow\left\|w_{0}\right\|_{\Omega}$ as $t \rightarrow 0$. This completes the proof.

Lemma 4.2. Assume $f \in L^{\infty}\left(0, T ; L^{2}(\Omega)\right)$ and $\partial_{t} f \in L^{\infty}\left(0, T ; L^{2}(\Omega)\right)$. Then there exists an integer $L \geq 1$ depending only on the discrete solution $U_{h}^{n-1}$ at time $t^{n-1}$, the mesh $\mathcal{M}^{n, 0}=\mathcal{M}^{n-1}$, the source function $f$, and the coefficient a $(x)$ such that $\tau_{n, k}=\tau_{n, L}$ for any $k \geq L$; that is, at each time step, the algorithm to determine the time-step size can be terminated in a finite number of steps.

Proof. Since $\partial_{t} f \in L^{\infty}\left(0, T ; L^{2}(\Omega)\right)$, we have

$$
\frac{1}{\tau_{n, k}} \int_{t^{n-1}}^{t^{n, k}}\left\|f-\bar{f}^{n, k}\right\|_{L^{2}(\Omega)} d t \leq \tau_{n, k} \sup _{0 \leq t \leq T}\left\|\frac{\partial f}{\partial t}\right\|_{L^{2}(\Omega)} .
$$

Thus for sufficiently small $\tau_{n, k}$, e.g.,

$$
\tau_{n, k} \leq \frac{1}{2 T} \sqrt{\operatorname{TOL}_{\text {time }}}\left(\sup _{0 \leq t \leq T}\left\|\frac{\partial f}{\partial t}\right\|_{L^{2}(\Omega)}\right)^{-1}
$$

the second inequality in (3.2) can be fulfilled. It remains to check the first inequality in (3.2). Let $\psi$ be the solution of the parabolic problem

$$
\begin{aligned}
& \frac{\partial \psi}{\partial t}-\operatorname{div}(a(x) \nabla \psi)=f \quad \text { in } \Omega \times\left(t^{n-1}, T\right), \\
& \psi=0 \quad \text { on } \Gamma \times\left(t^{n-1}, T\right), \quad \psi\left(x, t^{n-1}\right)=U_{h}^{n-1}(x) \quad \text { on } \Omega .
\end{aligned}
$$

Integrating the equation above in time from $t^{n-1}$ to $t^{n-1}+\tau_{n, k}$, we know that

$$
\left\langle\frac{\psi^{n, k}-U_{h}^{n-1}}{\tau_{n, k}}, \varphi\right\rangle+\left\langle a \nabla \bar{\psi}^{n, k}, \nabla \varphi\right\rangle=\left\langle\bar{f}^{n, k}, \varphi\right\rangle \quad \forall \varphi \in H_{0}^{1}(\Omega) .
$$

From Lemma 4.1 we have that for $\epsilon=\sqrt{3 \mathrm{TOL}_{\text {time }} / 2 T}$, there exists a constant $\tau>0$ such that as long as $\tau_{n, k} \leq \tau$, we have

$$
\left(\frac{1}{\tau_{n, k}}\left\|\psi^{n, k}-U_{h}^{n-1}\right\|_{L^{2}(\Omega)}^{2}+\left\|\bar{\psi}^{n, k}-U_{h}^{n-1}\right\|_{\Omega}^{2}\right)^{1 / 2} \leq \frac{\epsilon}{3} .
$$

Now for any given $\tau_{n, k}>0$, let $\hat{U}_{h}^{n, k} \in V_{0}^{n, 0}$ be the solution of the problem

$$
\left\langle\frac{\hat{U}_{h}^{n, k}-U_{h}^{n-1}}{\tau_{n, k}}, v\right\rangle+\left\langle a \nabla \hat{U}_{h}^{n, k}, \nabla v\right\rangle=\left\langle\bar{f}^{n, k}, v\right\rangle \quad \forall v \in V_{0}^{n, 0} .
$$

Since $\mathcal{M}^{n, k}$ is a refinement of $\mathcal{M}^{n, 0}$, we have $U_{h}^{n, k}-\hat{U}_{h}^{n, k} \in V_{0}^{n, k}$. Subtracting (4.5) from (4.1) and taking the test function as $U_{h}^{n, k}-\hat{U}_{h}^{n, k}$, we then have

$$
\left\langle\frac{\psi^{n, k}-U_{h}^{n, k}}{\tau_{n, k}}, U_{h}^{n, k}-\hat{U}_{h}^{n, k}\right\rangle+\left\langle a \nabla\left(\bar{\psi}^{n, k}-U_{h}^{n, k}\right), \nabla\left(U_{h}^{n, k}-\hat{U}_{h}^{n, k}\right)\right\rangle=0 .
$$


Using the identity $(a-c)^{2}=(a-b)^{2}-(b-c)^{2}+2(a-c)(b-c)$ for any $a, b, c \in \mathbf{R}$, we obtain

$$
\begin{aligned}
& \frac{1}{\tau_{n, k}}\left\|\psi^{n, k}-U_{h}^{n, k}\right\|_{L^{2}(\Omega)}^{2}+\left\|\bar{\psi}^{n, k}-U_{h}^{n, k}\right\|_{\Omega}^{2} \\
= & \frac{1}{\tau_{n, k}}\left\|\psi^{n, k}-\hat{U}_{h}^{n, k}\right\|_{L^{2}(\Omega)}^{2}+\left\|\bar{\psi}^{n, k}-\hat{U}_{h}^{n, k}\right\|_{\Omega}^{2} \\
- & \frac{1}{\tau_{n, k}}\left\|\hat{U}_{h}^{n, k}-U_{h}^{n, k}\right\|_{L^{2}(\Omega)}^{2}-\left\|\hat{U}_{h}^{n, k}-U_{h}^{n, k}\right\|_{\Omega}^{2} \\
\leq & \frac{1}{\tau_{n, k}}\left\|\psi^{n, k}-\hat{U}_{h}^{n, k}\right\|_{L^{2}(\Omega)}^{2}+\left\|\bar{\psi}^{n, k}-\hat{U}_{h}^{n, k}\right\|_{\Omega}^{2} .
\end{aligned}
$$

But, by the triangle inequality and (4.6), we have, for $\tau_{n, k} \leq \tau$,

$$
\begin{aligned}
& \left(\frac{1}{\tau_{n, k}}\left\|\psi^{n, k}-\hat{U}_{h}^{n, k}\right\|_{L^{2}(\Omega)}^{2}+\left\|\bar{\psi}^{n, k}-\hat{U}_{h}^{n, k}\right\|_{\Omega}^{2}\right)^{1 / 2} \\
\leq & \left(\frac{1}{\tau_{n, k}}\left\|\psi^{n, k}-U_{h}^{n-1}\right\|_{L^{2}(\Omega)}^{2}+\left\|\bar{\psi}^{n, k}-U_{h}^{n-1}\right\|_{\Omega}^{2}\right)^{1 / 2} \\
+ & \left(\frac{1}{\tau_{n, k}}\left\|\hat{U}_{h}^{n, k}-U_{h}^{n-1}\right\|_{L^{2}(\Omega)}^{2}+\left\|\hat{U}_{h}^{n, k}-U_{h}^{n-1}\right\|_{\Omega}^{2}\right)^{1 / 2} \\
\leq & \frac{\epsilon}{3}+\left(\frac{1}{\tau_{n, k}}\left\|\hat{U}_{h}^{n, k}-U_{h}^{n-1}\right\|_{L^{2}(\Omega)}^{2}+\left\|\hat{U}_{h}^{n, k}-U_{h}^{n-1}\right\|_{\Omega}^{2}\right)^{1 / 2} .
\end{aligned}
$$

Thus, combining (4.6), (4.8) and (4.9) and using the triangle inequality, we have, for $\tau_{n, k} \leq \tau$,

$$
\begin{aligned}
& \left\|U_{h}^{n, k}-U_{h}^{n-1}\right\|_{\Omega} \\
\leq & \left\|U_{h}^{n, k}-\bar{\psi}^{n, k}\right\|_{\Omega}+\left\|\bar{\psi}^{n, k}-U_{h}^{n-1}\right\|_{\Omega} \\
\leq & \frac{2}{3} \epsilon+\left(\frac{1}{\tau_{n, k}}\left\|\hat{U}_{h}^{n, k}-U_{h}^{n-1}\right\|_{L^{2}(\Omega)}^{2}+\left\|\hat{U}_{h}^{n, k}-U_{h}^{n-1}\right\|_{\Omega}^{2}\right)^{1 / 2} .
\end{aligned}
$$

It remains to estimate the error $\hat{U}_{h}^{n, k}-U_{h}^{n-1}$. From (4.7) we know that, for any $v \in V_{0}^{n, 0}$,

$$
\left\langle\frac{\hat{U}_{h}^{n, k}-U_{h}^{n-1}}{\tau_{n, k}}, v\right\rangle+\left\langle a \nabla\left(\hat{U}_{h}^{n, k}-U_{h}^{n-1}\right), \nabla v\right\rangle=\left\langle\bar{f}^{n, k}, v\right\rangle-\left\langle a \nabla U_{h}^{n-1}, \nabla v\right\rangle .
$$

Since $U_{h}^{n-1} \in V_{0}^{n-1}=V_{0}^{n, 0}$, we can take $v=\hat{U}_{h}^{n, k}-U_{h}^{n-1} \in V_{0}^{n, 0}$ in the identity above and obtain

$$
\begin{aligned}
& \frac{1}{\tau_{n, k}}\left\|\hat{U}_{h}^{n, k}-U_{h}^{n-1}\right\|_{L^{2}(\Omega)}^{2}+\left\|\hat{U}_{h}^{n, k}-U_{h}^{n-1}\right\|_{\Omega}^{2} \\
= & \left\langle\bar{f}^{n, k}, \hat{U}_{h}^{n, k}-U_{h}^{n-1}\right\rangle-\left\langle a \nabla U_{h}^{n-1}, \nabla\left(\hat{U}_{h}^{n, k}-U_{h}^{n-1}\right)\right\rangle .
\end{aligned}
$$

By the Cauchy-Schwarz inequality we have

$$
\begin{aligned}
\left\langle\bar{f}^{n, k}, \hat{U}_{h}^{n, k}-U_{h}^{n-1}\right\rangle & \leq\left\|\bar{f}^{n, k}\right\|_{L^{2}(\Omega)}\left\|\hat{U}_{h}^{n, k}-U_{h}^{n-1}\right\|_{L^{2}(\Omega)} \\
& \leq \frac{1}{4 \tau_{n, k}}\left\|\hat{U}_{h}^{n, k}-U_{h}^{n-1}\right\|_{L^{2}(\Omega)}^{2}+\tau_{n, k}\left\|\bar{f}^{n, k}\right\|_{L^{2}(\Omega)}^{2} .
\end{aligned}
$$


By the inverse estimate, we get

$$
\begin{aligned}
& \left\langle a \nabla U_{h}^{n-1}, \nabla\left(\hat{U}_{h}^{n, k}-U_{h}^{n-1}\right)\right\rangle \\
\leq & \sum_{K \in \mathcal{M}^{n, 0}} \bar{C} h_{K}^{-1}\left\|a \nabla U_{h}^{n-1}\right\|_{L^{2}(K)}\left\|\hat{U}_{h}^{n, k}-U_{h}^{n-1}\right\|_{L^{2}(K)} \\
\leq & \frac{1}{4 \tau_{n, k}}\left\|\hat{U}_{h}^{n, k}-U_{h}^{n-1}\right\|_{L^{2}(\Omega)}^{2}+\tau_{n, k} \bar{C}^{2} \sum_{K \in \mathcal{M}^{n, 0}} h_{K}^{-2}\left\|a \nabla U_{h}^{n-1}\right\|_{L^{2}(K)}^{2},
\end{aligned}
$$

where $\bar{C}$ is a constant depending on the minimum angle of $\mathcal{M}^{n, 0}$. Substituting the estimates above into (4.11), we finally get

$$
\begin{aligned}
& \frac{1}{2 \tau_{n, k}}\left\|\hat{U}_{h}^{n, k}-U_{h}^{n-1}\right\|_{L^{2}(\Omega)}^{2}+\left\|\hat{U}_{h}^{n, k}-U_{h}^{n-1}\right\|_{\Omega}^{2} \\
\leq & \tau_{n, k}\left(\|f\|_{L^{\infty}\left(0, T ; L^{2}(\Omega)\right)}^{2}+\bar{C}^{2} \sum_{K \in \mathcal{M}^{n, 0}} h_{K}^{-2}\left\|a \nabla U_{h}^{n-1}\right\|_{L^{2}(K)}^{2}\right)=: \tau_{n, k} \Delta_{n} .
\end{aligned}
$$

The quantity $\Delta_{n}$ depends only on $\mathcal{M}^{n, 0}, U_{h}^{n-1}, f(x, t)$, and $a(x)$, which are fixed through the adaptive procedure at the $n$-th time step. Thus, for sufficiently small $\tau_{n, k}$ (e.g., $\left.\tau_{n, k} \leq \epsilon^{2} /\left(18 \Delta_{n}\right)\right)$, we get

$$
\frac{1}{\tau_{n, k}}\left\|\hat{U}_{h}^{n, k}-U_{h}^{n-1}\right\|_{L^{2}(\Omega)}^{2}+\left\|\hat{U}_{h}^{n, k}-U_{h}^{n-1}\right\|_{\Omega}^{2} \leq 2 \tau_{n, k} \Delta_{n} \leq \frac{1}{9} \epsilon^{2}
$$

Substituting (4.13) into (4.10), we finally get that if $\tau_{n, k} \leq \min \left(\tau, \epsilon^{2} /\left(18 \Delta_{n}\right)\right)$, then

$$
\eta_{\text {time }}^{n, k}=\frac{1}{3}\left\|U_{h}^{n, k}-U_{h}^{n-1}\right\|_{\Omega}^{2} \leq \frac{1}{3} \epsilon^{2}=\frac{\text { TOL }_{\text {time }}}{2 T} .
$$

This completes the proof.

To conclude this subsection, we remark that the condition $\partial_{t} f \in L^{\infty}\left(0, T ; L^{2}(\Omega)\right)$ can be weakened. In fact, Lemma 4.2 holds if $f$ is piecewise smooth in time: $f$ has jumps at $0<T_{1}<T_{2}<\cdots<T_{I-1}<T$ and $\partial_{t} f \in L^{\infty}\left(T_{i-1}, T_{i} ; L^{2}(\Omega)\right)$. Here $T_{0}=0$ and $T_{I}=T$. In this case, we require additionally that $T_{i}, i=1,2 \ldots, I$ are the nodes of the time discretization; that is, $T_{i}=t^{n_{i}}$ for some $n_{i}$, where $t^{n}, n=0, \ldots, N$, is the partition of the time interval $[0, T]$.

4.2. Termination of space mesh refinements. Now we turn to the finite step termination of mesh refinements. By Lemma 4.2 we can, without loss of generality, fix the time-step size $\tau_{n, k}=\tau_{n, L}$. In the following we will write $\tau_{n, k}=\tau_{n}$. Let $\hat{C}_{n, k}=\max \left\{h_{K}^{2} / \tau_{n}: K \in \mathcal{M}^{n, k}\right\}$. Note that $\hat{C}_{n, k+1} \leq \hat{C}_{n, k}$ if $\mathcal{M}^{n, k+1}$ is a refinement of $\mathcal{M}^{n, k}$. Thus there exists a constant $\tilde{C}_{n}$ which is fixed in the procedure for doing mesh refinements such that

$$
\hat{C}_{n, k} \leq \tilde{C}_{n} \quad \forall k \geq L .
$$

To begin with, we first introduce the following refinement strategy by Morin, Nochetto and Siebert [15] for linear elliptic problems. This strategy is an improvement of the Guaranteed Convergence Strategy of Dörfler [11.

MNS Refinement Strategy. Given two parameters $0<\theta, \hat{\theta}<1$, mesh $\mathcal{M}^{n, k}$, and solution $U_{h}^{n, k} \in V_{0}^{n, k}$ over the mesh $\mathcal{M}^{n, k}$, 
1. Select a subset $\hat{\mathcal{B}}^{n, k}$ of sides in $\mathcal{B}^{n, k}$ such that

$$
\left(\sum_{e \in \hat{\mathcal{B}}^{n, k}} \eta_{e}^{n, k}\right)^{1 / 2} \geq \theta\left(\sum_{e \in \mathcal{B}^{n, k}} \eta_{e}^{n, k}\right)^{1 / 2}
$$

2. Let $\hat{\mathcal{M}}^{n, k}$ be the set of elements with one side in $\hat{\mathcal{B}}^{n, k}$. Enlarge $\hat{\mathcal{M}}^{n, k}$ such that

$$
\operatorname{osc}\left(R^{n, k}, \hat{\mathcal{M}}^{n, k}\right) \geq \hat{\theta} \operatorname{osc}\left(R^{n, k}, \mathcal{M}^{n, k}\right) .
$$

3. Refine every element in $\hat{\mathcal{M}}^{n, k}$ in such a way that a node is created in the interior of the element.

Here $\mathcal{B}^{n, k}$ is the collection of all interior interelement sides, $\eta_{e}^{n, k}$ is the local space error indicator associated with $e \in \mathcal{B}^{n, k}$

$$
\eta_{e}^{n, k}=\frac{1}{2} \sum_{K \in \Omega_{e}} h_{K}^{2}\left\|R^{n, k}\right\|_{L^{2}(K)}^{2}+h_{e}\left\|J_{e}^{n, k}\right\|_{L^{2}(e)}^{2}
$$

with $R^{n, k}=\bar{f}^{n}-\left(U_{h}^{n, k}-U_{h}^{n-1}\right) / \tau_{n}, J_{e}^{n, k}=\llbracket a \nabla U_{h}^{n, k} \rrbracket_{e} \cdot \nu_{e}$, and $\Omega_{e}$ is the collection of two elements sharing $e$ as a common side. The oscillations $\operatorname{osc}\left(R^{n, k}, \hat{\mathcal{M}}^{n, k}\right)$ and $\operatorname{osc}\left(R^{n, k}, \mathcal{M}^{n, k}\right)$ are defined as in (1.5). For a discussion on the choices of the parameters $\theta, \hat{\theta}$ and their influence on the performance of adaptive methods, we refer to [6]. Usually, a smaller $\theta$ leads to better performance.

The starting point of the analysis is the following orthogonality result whose proof is similar to that of (3.7) in Theorem 3.1

Lemma 4.3. If $\mathcal{M}^{n, k+1}$ is a refinement of $\mathcal{M}^{n, k}$ such that $V^{n, k} \subset V^{n, k+1}$, then the relation

$$
\left\|U_{*}^{n}-U_{h}^{n, k+1}\right\|_{\tau_{n}, \Omega}^{2}=\left\|U_{*}^{n}-U_{h}^{n, k}\right\|_{\tau_{n}, \Omega}^{2}-\left\|U_{h}^{n, k}-U_{h}^{n, k+1}\right\|_{\tau_{n}, \Omega}^{2}
$$

holds. Here $\|\cdot\|_{\tau_{n}, \Omega}$ is defined in (1.7).

For any $K \in \mathcal{M}^{n, k}$ and $\varphi \in L^{2}(\Omega)$, define $P_{K} \varphi=\frac{1}{|K|} \int_{K} \varphi d x$. The following lemma can be proved exactly as in [15] Lemma 4.2] by using a similar idea as in the proof of Theorem 2.2. Here we omit the details.

Lemma 4.4. Let $\mathcal{M}^{n, k+1}$ be a refinement of $\mathcal{M}^{n, k}$ according to the MNS Refinement Strategy. Then there exist constants $C_{4}, C_{5}>0$, depending only on the minimum angle of $\mathcal{M}^{n, k}$ and on the coefficient $a(x)$ such that, for all $e \in \hat{\mathcal{B}}^{n, k}$, we have

$$
\eta_{e}^{n, k} \leq C_{4} \hat{C}_{n, k} \sum_{K \in \Omega_{e}}\left\|U_{h}^{n, k+1}-U_{h}^{n, k}\right\|_{\tau_{n}, K}^{2}+C_{5} \sum_{K \in \Omega_{e}} h_{K}^{2}\left\|R^{n, k}-P_{K} R^{n, k}\right\|_{L^{2}(K)}^{2},
$$

where $\hat{C}_{n, k}$ is defined at the beginning of this subsection.

Now since $U_{h}^{n, k} \in V_{0}^{n, k}$ is the solution of the discrete problem

$$
\left\langle\frac{U_{h}^{n, k}-U_{h}^{n-1}}{\tau_{n}}, v\right\rangle+\left\langle a \nabla U_{h}^{n, k}, \nabla v\right\rangle=\left\langle\bar{f}^{n}, v\right\rangle \quad \forall v \in V_{0}^{n, k},
$$

which is an approximation of equation (1.4), we deduce by the standard a posteriori error analysis that there exists a constant $C_{1}>0$ such that

$$
\left\|U_{*}^{n}-U_{h}^{n, k}\right\|_{\tau_{n}, \Omega}^{2} \leq C_{1} \sum_{e \in \mathcal{B}^{n, k}} \eta_{e}^{n, k}
$$


From Step 1 in the MNS Refinement Strategy, we know that

$$
\theta^{2}\left\|U_{*}^{n}-U_{h}^{n, k}\right\|_{\tau_{n}, \Omega}^{2} \leq \theta^{2} C_{1} \sum_{e \in \mathcal{B}^{n, k}} \eta_{e}^{n, k} \leq C_{1} \sum_{e \in \hat{\mathcal{B}}^{n, k}} \eta_{e}^{n, k}
$$

which yields, upon using Lemma 4.4 and (4.14),

$$
\begin{aligned}
\theta^{2}\left\|U_{*}^{n}-U_{h}^{n, k}\right\|_{\tau_{n}, \Omega}^{2} & \leq 2 C_{1} C_{4} \tilde{C}_{n}\left\|U_{h}^{n, k+1}-U_{h}^{n, k}\right\|_{\tau_{n}, \Omega}^{2} \\
& +2 C_{1} C_{5} \operatorname{osc}\left(R^{n, k}, \mathcal{M}^{n, k}\right)^{2} .
\end{aligned}
$$

Let $\gamma \in(0,1)$ be the reduction factor of element size associated with one refinement step. Given $\hat{\theta} \in(0,1)$, let $\hat{\alpha}=\left(1-\left(1-\gamma^{2}\right) \hat{\theta}^{2}\right)^{1 / 2}<1$. Then by Step 2 in the MNS Refinement Strategy we obtain, as in [15, Lemma 3.8], that

$$
\operatorname{osc}\left(R^{n, k}, \mathcal{M}^{n, k+1}\right) \leq \hat{\alpha} \operatorname{osc}\left(R^{n, k}, \mathcal{M}^{n, k}\right) .
$$

On the other hand, since

$$
R^{n, k+1}=\bar{f}^{n}-\frac{U_{h}^{n, k+1}-U_{h}^{n-1}}{\tau_{n}}=R^{n, k}-\frac{U_{h}^{n, k+1}-U_{h}^{n, k}}{\tau_{n}},
$$

we deduce from Young's inequality and (4.16) that

$$
\begin{aligned}
& \operatorname{osc}\left(R^{n, k+1}, \mathcal{M}^{n, k+1}\right)^{2} \\
\leq & (1+\delta) \operatorname{osc}\left(R^{n, k}, \mathcal{M}^{n, k+1}\right)^{2}+\left(1+\delta^{-1}\right) \operatorname{osc}\left(\frac{U_{h}^{n, k+1}-U_{h}^{n, k}}{\tau_{n}}, \mathcal{M}^{n, k+1}\right)^{2} \\
\leq & \hat{\alpha}^{2}(1+\delta) \operatorname{osc}\left(R^{n, k}, \mathcal{M}^{n, k}\right)^{2}+\left(1+\delta^{-1}\right) \operatorname{osc}\left(\frac{U_{h}^{n, k+1}-U_{h}^{n, k}}{\tau_{n}}, \mathcal{M}^{n, k+1}\right)^{2},
\end{aligned}
$$

where $\delta>0$ is some constant such that $\hat{\alpha}^{2}(1+\delta)<1$.

Lemma 4.5. Set $\zeta_{1}=\hat{\alpha}^{2}(1+\delta)<1$ and $\zeta_{2}=\left(1+\delta^{-1}\right) \tilde{C}_{n}$. Let $\alpha, \beta \in(0,1)$ be the constants defined by

$$
\beta=1-\frac{2}{2+\left(1-\zeta_{1}\right)\left(1+\delta^{-1}\right)^{-1} C_{4} C_{5}^{-1}}, \quad \alpha=\left(1-\frac{\theta^{2} \beta}{2 C_{1} C_{4} \tilde{C}_{n}}\right)^{1 / 2} .
$$

Then if $\mathcal{M}^{n, k+1}$ is a refinement of $\mathcal{M}^{n, k}$ according to the MNS Refinement Strategy, the following estimate is valid:

$$
\begin{aligned}
& \left\|U_{*}^{n}-U_{h}^{n, k+1}\right\|_{\tau_{n}, \Omega}^{2}+(1-\beta) \zeta_{2}^{-1} \operatorname{osc}\left(R^{n, k+1}, \mathcal{M}^{n, k+1}\right)^{2} \\
\leq & \max \left(\alpha^{2}, \frac{1}{2}\left(1+\zeta_{1}\right)\right)\left(\left\|U_{*}^{n}-U_{h}^{n, k}\right\|_{\tau_{n}, \Omega}^{2}+(1-\beta) \zeta_{2}^{-1} \operatorname{osc}\left(R^{n, k}, \mathcal{M}^{n, k}\right)^{2}\right) .
\end{aligned}
$$

Proof. First we note that

$$
\begin{aligned}
& \operatorname{osc}\left(\frac{U_{h}^{n, k+1}-U_{h}^{n, k}}{\tau_{n}}, \mathcal{M}^{n, k+1}\right)^{2} \\
= & \sum_{K \in \mathcal{M}^{n, k+1}} h_{K}^{2} \tau_{n}^{-2}\left\|\left(U_{h}^{n, k+1}-U_{h}^{n, k}\right)-P_{K}\left(U_{h}^{n, k+1}-U_{h}^{n, k}\right)\right\|_{L^{2}(K)}^{2} \\
\leq & \hat{C}_{n, k+1} \sum_{K \in \mathcal{M}^{n, k+1}} \tau_{n}^{-1}\left\|U_{h}^{n, k+1}-U_{h}^{n, k}\right\|_{L^{2}(K)}^{2} \\
\leq & \tilde{C}_{n} \tau_{n}^{-1}\left\|U_{h}^{n, k+1}-U_{h}^{n, k}\right\|_{L^{2}(\Omega)}^{2} \\
\leq & \tilde{C}_{n}\left\|U_{h}^{n, k+1}-U_{h}^{n, k}\right\|_{\tau_{n}, \Omega}^{2} .
\end{aligned}
$$


Thus, by (4.17) we get

(4.18) $\operatorname{osc}\left(R^{n, k+1}, \mathcal{M}^{n, k+1}\right)^{2} \leq \zeta_{1} \operatorname{osc}\left(R^{n, k}, \mathcal{M}^{n, k}\right)^{2}+\zeta_{2}\left\|U_{h}^{n, k+1}-U_{h}^{n, k}\right\|_{\tau_{n}, \Omega}^{2}$.

From (4.15) we obtain

$\left\|U_{h}^{n, k+1}-U_{h}^{n, k}\right\|_{\tau_{n}, \Omega}^{2} \geq \frac{\theta^{2}}{2 C_{1} C_{4} \tilde{C}_{n}}\left\|U_{*}^{n}-U_{h}^{n, k}\right\|_{\tau_{n}, \Omega}^{2}-\frac{C_{5}}{C_{4} \tilde{C}_{n}} \operatorname{osc}\left(R^{n, k}, \mathcal{M}^{n, k}\right)^{2}$,

which, together with Lemma 4.3, yields

$$
\begin{aligned}
\| U_{*}^{n}- & U_{h}^{n, k+1} \|_{\tau_{n}, \Omega}^{2} \\
= & \left\|U_{*}^{n}-U_{h}^{n, k}\right\|_{\tau_{n}, \Omega}^{2} \\
& -\beta\left\|U_{h}^{n, k+1}-U_{h}^{n, k}\right\|_{\tau_{n}, \Omega}^{2}-(1-\beta)\left\|U_{h}^{n, k+1}-U_{h}^{n, k}\right\|_{\tau_{n}, \Omega}^{2} \\
\leq & \alpha^{2}\left\|U_{*}^{n}-U_{h}^{n, k}\right\|_{\tau_{n}, \Omega}^{2} \\
& +\beta \frac{C_{5}}{C_{4} \tilde{C}_{n}} \operatorname{osc}\left(R^{n, k}, \mathcal{M}^{n, k}\right)^{2}-(1-\beta)\left\|U_{h}^{n, k+1}-U_{h}^{n, k}\right\|_{\tau_{n}, \Omega}^{2} .
\end{aligned}
$$

Multiplying (4.18) by $\zeta_{2}^{-1}(1-\beta)$ and adding it to the estimate above, we get

$$
\begin{aligned}
& \left\|U_{*}^{n}-U_{h}^{n, k+1}\right\|_{\tau_{n}, \Omega}^{2}+(1-\beta) \zeta_{2}^{-1} \operatorname{osc}\left(R^{n, k+1}, \mathcal{M}^{n, k+1}\right)^{2} \\
\leq & \alpha^{2}\left\|U_{*}^{n}-U_{h}^{n, k}\right\|_{\tau_{n}, \Omega}^{2}+\left(\beta C_{5} C_{4}^{-1} \tilde{C}_{n}^{-1}+(1-\beta) \zeta_{1} \zeta_{2}^{-1}\right) \operatorname{osc}\left(R^{n, k}, \mathcal{M}^{n, k}\right)^{2} \\
\leq & \max \left(\alpha^{2}, \mu\right)\left(\left\|U_{*}^{n}-U_{h}^{n, k}\right\|_{\tau_{n}, \Omega}^{2}+(1-\beta) \zeta_{2}^{-1} \operatorname{osc}\left(R^{n, k}, \mathcal{M}^{n, k}\right)^{2}\right),
\end{aligned}
$$

where

$$
\mu=\frac{\beta C_{5} C_{4}^{-1} \tilde{C}_{n}^{-1}+(1-\beta) \zeta_{1} \zeta_{2}^{-1}}{(1-\beta) \zeta_{2}^{-1}}=\zeta_{1}+\frac{\beta}{1-\beta}\left(1+\delta^{-1}\right) C_{5} C_{4}^{-1} .
$$

This completes the proof by observing that $\frac{\beta}{1-\beta}=\frac{1}{2}\left(1-\zeta_{1}\right)\left(1+\delta^{-1}\right)^{-1} C_{4} C_{5}^{-1}$.

Now by the lower bound (2.10) proved in $\S 2.2$ and using (4.14), we know that

$$
\begin{aligned}
\eta_{\text {space }}^{n, k+1} & \leq 2 C_{2} \hat{C}_{n, k+1}\left\|U_{*}^{n}-U_{h}^{n, k+1}\right\|_{\tau_{n}, \Omega}^{2}+2 C_{3} \operatorname{osc}\left(R^{n, k+1}, \mathcal{M}^{n, k+1}\right)^{2} \\
& \leq 2 C_{2} \tilde{C}_{n}\left\|U_{*}^{n}-U_{h}^{n, k+1}\right\|_{\tau_{n}, \Omega}^{2}+2 C_{3} \operatorname{osc}\left(R^{n, k+1}, \mathcal{M}^{n, k+1}\right)^{2} .
\end{aligned}
$$

Since $\alpha^{2}<1$ and $\frac{1}{2}\left(1+\zeta_{1}\right)<1$, we conclude from Lemma 4.5 that

$$
\lim _{k \rightarrow \infty}\left\|U_{*}^{n}-U_{h}^{n, k}\right\|_{\tau_{n}, \Omega}=0, \quad \lim _{k \rightarrow \infty} \operatorname{osc}\left(R^{n, k}, \mathcal{M}^{n, k}\right)=0 .
$$

Thus $\eta_{\text {space }}^{n, k} \rightarrow 0$ as $k \rightarrow \infty$. This together with Lemma 4.2 implies the following theorem, which is the main result of this section.

Theorem 4.6. Assume that $f \in L^{\infty}\left(0, T ; L^{2}(\Omega)\right)$ and $\partial_{t} f \in L^{\infty}\left(0, T ; L^{2}(\Omega)\right)$. Then, at each time step $n \geq 1$, if the MNS Refinement Strategy is used in doing mesh refinements, Algorithm 3.2 will stop in a finite number of steps for any given tolerances $\mathrm{TOL}_{\mathrm{time}}, \mathrm{TOL}$ space and $\mathrm{TOL}$ coarse.

Remark 4.7. The following observation, which is made to us by the referee of the paper, points out an important consequence of the analysis in this section on the choice of the coarsening tolerance and the time tolerance. If the exact solution has a positive energy and is within a good tolerance from the approximate solution, then 
$\left\|a \nabla U_{h}^{n-1}\right\|_{L^{2}(\Omega)}^{2} \geq c_{0}$ for some positive constant $c_{0}$. It follows from the definition of $\Delta_{n}$ in (4.12) that

$$
\Delta_{n} \geq \frac{c_{0} \bar{C}^{2}}{h_{n, 0}^{2}}, \quad \text { where } h_{n, 0}=\max _{K \in \mathcal{M}^{n, 0}} h_{K} .
$$

This yields, from the proof of Lemma 4.2 that

$$
\tau_{n}=\tau_{n, L} \leq \frac{\epsilon^{2}}{18 \Delta_{n}} \leq \frac{\epsilon^{2} h_{n, 0}^{2}}{18 c_{0} \bar{C}^{2}} \leq \frac{h_{n, 0}^{2}}{c_{0} \bar{C}^{2} T} \text { TOL }_{\text {time }} .
$$

On the other hand, according to the definition of $\hat{C}_{n, 0}$ at the beginning of $\S 4.2$, we have

$$
h_{n, 0}^{2} \leq \hat{C}_{n-1} \tau_{n-1} .
$$

This implies that the constant $\hat{C}_{H}^{m}$ in Theorem 3.2 satisfies

$$
\hat{C}_{H}^{m}=\max _{n \leq m+1} \hat{C}_{n-1} \geq \frac{c_{0} \bar{C}^{2} T}{\mathrm{TOL}_{\text {time }}} \max _{n \leq m+1} \frac{\tau_{n}}{\tau_{n-1}} \geq \frac{c_{0} \bar{C}^{2} T}{\mathrm{TOL}_{\text {time }}},
$$

whenever $\max _{n \leq m+1}\left(\tau_{n} / \tau_{n-1}\right) \geq 1$. Notice that if the global algorithm is ever to terminate, that is, to reach time $T$ with the estimated error below the prescribed tolerances, then it is necessary that $\max \left(\tau_{n} / \tau_{n-1}\right) \geq 1$. A comparison of (4.19) with estimate (3.9) implies that the ratio between the coarsening tolerance and the time tolerance has to be small. In other words, a small time tolerance puts a severe restriction on the coarsening tolerance if the overall error has to be small.

We also remark that Theorem 4.6] states that Algorithm 3.2 can be terminated in a finite number of iteration steps at any time step $t^{n}$ starting from any given solution $U_{h}^{n-1}$ at $t^{n-1}$. Whether the adaptive algorithm can reach the final time $T$ is an open question which is not addressed in this paper and requires further research.

\section{Numerical EXPERIMENT}

The implementation of Algorithm 3.2 is based on a 2D adaptive finite element solver for elliptic problems developed by Shibin Dai. The MNS Refinement Strategy is implemented as in [15, Algorithm 5.1]. We use the method indicated in Figure 1] to refine a marked element which creates an interior node.

Now we turn to the algorithm for mesh coarsening in Step 4 in Algorithm 3.2 Let $K$ be some element which is obtained by dividing some parent element $\hat{K}$ into two elements using the bisection algorithm. Denote by $\hat{e}$ the edge of $\hat{K}$ which is divided, and $\hat{K}^{\prime}$ the neighboring macro-element sharing the common edge $\hat{e}$ with
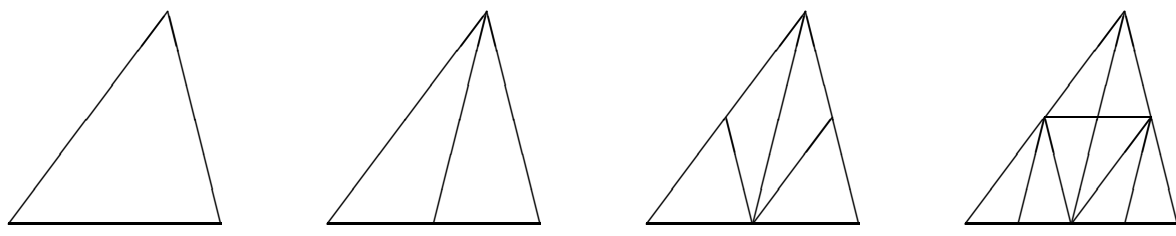

Figure 1. Refinement of a marked triangle which generates an interior node 
$\hat{K}$. We define the patch of elements $\mathcal{P}(K)$ associated with $K$ as the collection of all subelements included in $\hat{K}$ and $\hat{K}^{\prime}$. We note that if $K$ is marked for coarsening, then all elements in $\mathcal{P}(K)$ must also be marked for coarsening. For $n \geq 1$, we let $\mathcal{M}^{n}$ be the mesh obtained after doing the first three steps in Algorithm 3.2 which now is ready for possible coarsening, and $U_{h}^{n}$ be the corresponding discrete solution. The elements in $\mathcal{M}^{n}$ which are obtained by refining some element (possibly many times) in $\mathcal{M}^{n, 0}=\mathcal{M}^{n-1}$ are also not allowed for coarsening. All the elements in $\mathcal{M}^{n}$ which can be coarsened are called admissible coarsening elements. The collection of all admissible coarsening elements is denoted by $\hat{\mathcal{M}}^{n}$. For any $K \in \hat{\mathcal{M}}^{n}$, we denote by $\hat{K}$ its parent element such that $K$ is obtained by refining $\hat{K}$ into two elements using the bisection algorithm. Then for each $K \in \hat{\mathcal{M}}^{n}$, we can compute the local coarsening error indicator as follows:

$$
\eta_{\text {coarse }, K}^{n}=\frac{1}{\tau_{n}}\left\|U_{h}^{n}-\hat{U}_{h}^{n}\right\|_{L^{2}(K)}^{2}+\left\|U_{h}^{n}-\hat{U}_{h}^{n}\right\|_{K}^{2},
$$

where $\hat{U}_{h}^{n}$ is the linear function on $\hat{K}$ whose values at the vertexes of $\hat{K}$ are the same as those of $U_{h}^{n}$. The guideline of the coarsening strategy is then to mark as many elements $K \in \hat{\mathcal{M}}^{n}$ as possible so that

$$
\sum_{K \text { marked }} \eta_{\text {coarse }, K}^{n} \leq \frac{\text { TOL }_{\text {coarse }}}{T}
$$

is satisfied. In a practical situation, it may happen that after all elements in $\hat{\mathcal{M}}^{n}$ marked for coarsening have been coarsened, the coarsening error is still below the tolerance; that is,

$$
\eta_{\text {coarse }}^{n, 1}=\frac{1}{\tau_{n}}\left\|U_{h}^{n}-I_{H}^{n, 1} U_{h}^{n}\right\|_{L^{2}(\Omega)}^{2}+\left\|U_{h}^{n}-I_{H}^{n, 1} U_{h}^{n}\right\|_{\Omega}^{2}<\frac{\mathrm{TOL}_{\text {coarse }}}{T},
$$

where $\hat{\mathcal{M}}_{H}^{n, 1}$ is the mesh produced by coarsening all the elements in $\mathcal{M}^{n}$ marked for coarsening, and $I_{H}^{n, 1}$ is the associated linear finite element interpolant over $\mathcal{M}_{H}^{n, 1}$. If $\mathcal{M}_{H}^{n}$ is a further coarsening of $\mathcal{M}_{H}^{n, 1}$, then by the triangle inequality we have

$$
\begin{aligned}
\left\|U_{h}^{n}-I_{H}^{n} U_{h}^{n}\right\|_{\tau_{n}, \Omega} & \leq\left\|U_{h}^{n}-I_{H}^{n, 1} U_{h}^{n}\right\|_{\tau_{n}, \Omega}+\left\|I_{H}^{n, 1} U_{h}^{n}-I_{H}^{n} U_{h}^{n}\right\|_{\tau_{n}, \Omega} \\
& =\left\|U_{h}^{n}-I_{H}^{n, 1} U_{h}^{n}\right\|_{\tau_{n}, \Omega}+\left\|I_{H}^{n, 1} U_{h}^{n}-I_{H}^{n}\left(I_{H}^{n, 1} U_{h}^{n}\right)\right\|_{\tau_{n}, \Omega} .
\end{aligned}
$$

Thus in the case of (5.1) we can set $\mathcal{M}^{n}=\mathcal{M}_{H}^{n, 1}, U_{h}^{n}=I_{H}^{n, 1} U_{h}^{n}$ and start the mesh coarsening iteration again. The algorithm to coarsen the mesh is terminated when either $\eta_{\text {coarse }}^{n} \leq \mathrm{TOL}_{\text {coarse }} / T$ is violated or the marked coarsening set of $\mathcal{M}^{n}$ is empty. The following algorithm provides one possible implementation of the coarsening strategy.

Algorithm 5.1 (Mesh coarsening). Prescribe the tolerance TOL $\mathrm{L}_{\text {coarse }}$, the parameter $\nu \in(0,1)$, the mesh $\mathcal{M}^{n}$, and the solution $U_{h}^{n}$ over the mesh $\mathcal{M}^{n}$.

set $\sigma_{1}=0, \sigma_{2}=0, \gamma=0, \rho=\mathrm{TOL}_{\text {coarse }} / 4 T$

if $\sigma_{2}<\rho$ do

form the set $\hat{\mathcal{M}}^{n}$ of admissible coarsening elements compute $\eta_{\text {coarse }, K}^{n}$ for any $K \in \hat{\mathcal{M}}^{n}$

$\eta_{\max }=\max \left\{\eta_{\text {coarse }, K}^{n}: K \in \hat{\mathcal{M}}^{n}\right\}$

while $\gamma \leq 1$ and $\eta_{\max }>0$ do

$$
\gamma=\gamma+\nu
$$


for all $K$ in $\hat{\mathcal{M}}^{n}$ do

if $K$ is not marked for coarsening

$$
\begin{aligned}
& \text { if } \eta_{\text {coarse }, K}^{n} \leq \gamma \eta_{\max } \text { and } K^{\prime} \in \hat{\mathcal{M}}^{n} \text { for any } K^{\prime} \in \mathcal{P}(K) \\
& \sigma_{2}=\sigma_{2}+\sum_{K^{\prime} \in \mathcal{P}(K)} \eta_{\text {coarse }, K^{\prime}}^{n} \\
& \text { if } \sigma_{2} \leq \rho \\
& \text { mark all elements in } \mathcal{P}(K) \text { for coarsening }
\end{aligned}
$$
else

$$
\sigma_{2}=\sigma_{2}-\sum_{K^{\prime} \in \mathcal{P}(K)} \eta_{\text {coarse }, K^{\prime}}^{n}
$$

end if end if

end if

end for

end while

set $\sigma_{1}=\sigma_{1}+\sqrt{\sigma_{2}}, \sigma_{2}=0$

if $\eta_{\max }=0$ do

mark all elements in $\hat{\mathcal{M}}^{n}$ for coarsening

\section{end if}

set $\mathcal{M}^{n}$ as the mesh obtained by coarsening all elements marked for coarsening

set $U_{h}^{n}$ as the linear interpolant of $U_{h}^{n}$ on the coarsened mesh

if the number of elements before and after the coarsening is the same

end if go to end

$\rho=\max \left(0, \sqrt{\mathrm{TOL}_{\text {coarse }} / T}-\sigma_{1}\right)^{2}$

end if

if end

Now we report a numerical example computed by using Algorithm 3.2 with the MNS Refinement Strategy and Algorithm [5.1] We take the parameters $\delta_{1}=$

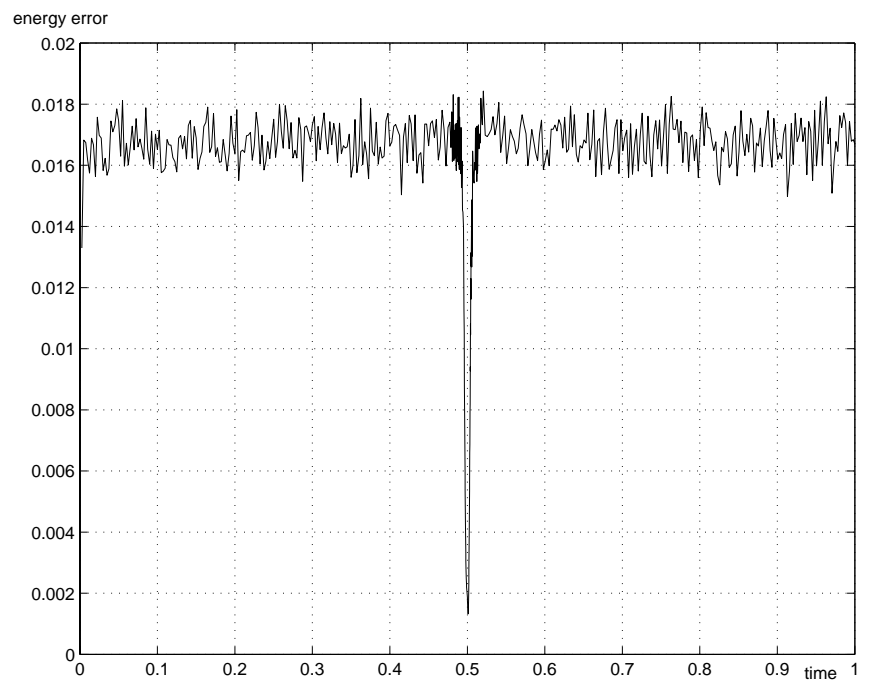

FIGURE 2. The energy error at each time step when $\mathrm{TOL}_{\text {space }}=0.01$. 
TABLE 5.1. The number of time steps $N$, the average number of nodes of the meshes av $\left(\mathcal{M}^{n}\right)$, the total estimated error $\eta$, the total energy error $\mathcal{E}$, and the effectiveness index eff for different values of $\mathrm{TOL}_{\text {space }}$.

\begin{tabular}{|c|c|c|c|c|c|}
\hline TOL $_{\text {space }}$ & $N$ & $\mathrm{av}\left(\mathcal{M}^{n}\right)$ & $\eta$ & $\mathcal{E}$ & eff \\
\hline \hline 0.04 & 257 & 297 & $2.095 E-1$ & $3.235 E-2$ & 6.4740 \\
\hline 0.01 & 512 & 786 & $1.057 E-1$ & $1.671 E-2$ & 6.3260 \\
\hline 0.0025 & 1028 & 2736 & $5.300 E-2$ & $8.470 E-3$ & 6.2613 \\
\hline \hline 0.02 & 292 & 441 & $1.580 E-1$ & $2.313 E-2$ & 6.8326 \\
\hline 0.005 & 587 & 1337 & $7.971 E-2$ & $1.200 E-2$ & 6.6421 \\
\hline 0.00125 & 1175 & 4912 & $3.985 E-2$ & $6.058 E-3$ & 6.5784 \\
\hline
\end{tabular}

$0.5, \delta_{2}=2, \theta_{\text {time }}=0.5$ in Algorithm 3.2, $\theta=0.2, \hat{\theta}=0.1$ in the MNS Refinement Strategy, and $\nu=0.05$ in Algorithm 5.1. We set $\Omega=(-1,1) \times(-1,1)$ and $T=1$. The exact solution of the continuous problem is prescribed as

$$
u(x, t)=\beta(t) * e^{-\left[\left(x_{1}-t+0.5\right)^{2}+\left(x_{2}-t+0.5\right)^{2}\right] / 0.04}
$$

with

$$
\beta(t)=0.1 *\left(1-e^{-10000 *(t-0.5)^{2}}\right) .
$$

We observe that $\beta(t)$ drops exponentially around $t=0.5$. The tolerances used are

$$
\mathrm{TOL}_{\text {time }}=\mathrm{TOL}_{\text {space }}, \quad \mathrm{TOL}_{\text {coarse }}=0.03 * \mathrm{TOL}_{\text {space }} .
$$

The effectiveness index eff of the a posteriori error estimate is defined as eff $=$ $\eta / \mathcal{E}$, where $\mathcal{E}=\left(\sum_{n=1}^{N} \tau_{n}\left\|u^{n}-U_{h}^{n}\right\|_{\Omega}^{2}\right)^{1 / 2}$ is the total energy error and $\eta$ is the estimated error

$$
\eta=\left\{\sum_{n=1}^{N} \tau_{n}\left(\eta_{\text {space }}^{n}+\eta_{\text {time }}^{n}+\eta_{\text {coarse }}^{n}\right)+2\left(\sum_{n=1}^{m} \int_{t^{n-1}}^{t^{n}}\left\|f-\bar{f}^{n}\right\|_{L^{2}(\Omega)}\right)^{2}\right\}^{1 / 2} .
$$
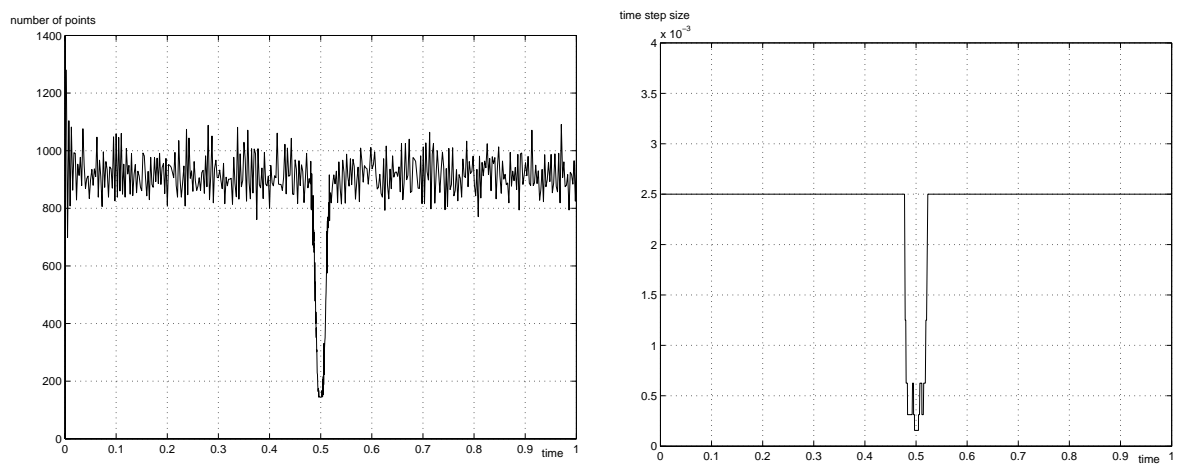

Figure 3. The number of nodes of $\mathcal{M}^{n}$ and the time-step size $\tau_{n}$ at each time step $n$ when $\mathrm{TOL}_{\text {space }}=0.01$. 

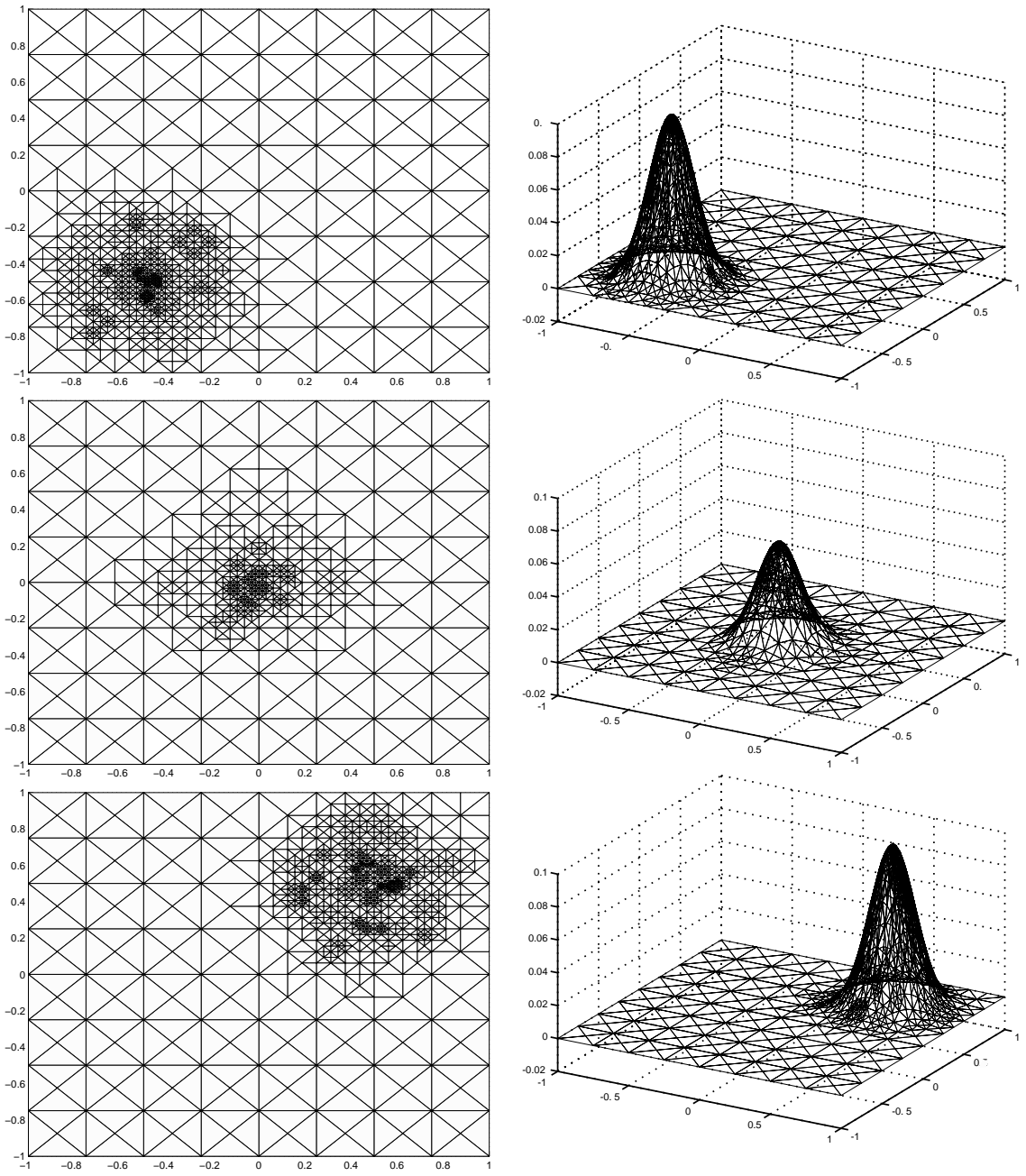

FiguRE 4. Surface plots of discrete solutions (right) and the corresponding meshes (left) at time $t=0.01,0.49,0.998125$ (from top to bottom). The numbers of nodes of the meshes are $808,459,825$ for $t=0.01,0.49,0.998125$, respectively.

In Table 5.1 we report the number of time steps $N$, the average number of nodes of the meshes $\operatorname{av}\left(\mathcal{M}^{n}\right)$, the estimated error $\eta$, the total energy error $\mathcal{E}$, and the effectiveness index eff, when running the algorithm for different values of $\mathrm{TOL}_{\text {space }}$. Recall from Theorem 3.2 (neglecting the initial error) that $\mathcal{E}^{2} \leq C \mathrm{TOL}_{\text {space }}$ due to Theorem 3.2 and (15.2). Thus by reducing the tolerance $\mathrm{TOL}_{\text {space }}$ by one-fourth we are actually requiring the reduction of the total energy error $\mathcal{E}$ by half, which is indeed observed in Table 5.1. Moreover, we observe that when the error $\mathcal{E}$ is reduced by half, the number of time steps is increased by roughly twice, and the average number of nodes of the meshes is increased less than fourth. Most interestingly, the effectiveness indexes eff remain roughly constant in the computations. 
Now we show more computational results when $\mathrm{TOL}_{\text {space }}=0.01$. The energy error $\left\|u^{n}-U_{h}^{n}\right\|_{\Omega}$ at each time step $n$ is depicted in Figure 2 The number of nodes and the time-step size at each time step $n$ are shown in Figure [3. We note that the time-step size drops in a small time interval around $t=0.5$ and is constant away from this interval, which is not surprising as $\beta(t)$ changes exponentially from 0.1 to 0 and then from 0 to 0.1 around $t=0.5$, and away from $t=0.5, \beta \approx 0.1$ and the peak of the exact solution moves at constant speed and the shape of the solution remains unchanged. This also explains why the energy errors and the numbers of nodes of the meshes are smaller around $t=0.5$ as the exact solution $u$ is smaller around $t=0.5$. The surface plots of the computed solution and the associated adaptive mesh at time $t=0.01,0.49,0.998125$ are displayed in Figure 4 which clearly indicates the ability of Algorithm 3.2 to capture the singularity of the solutions by locally refining and coarsening the meshes.

\section{ACKNOWLEDGMENT}

The authors would like to thank the referee for acquainting us with the work of Picasso [17] and for several constructive comments.

\section{REFERENCES}

1. E. Bänsch, Local mesh refinement in 2 and 3 dimensions, IMPACT Comput. Sci. Engrg. 3 (1991), 181-191. MR 92h:65150

2. M. Bieterman and I. Babuška, The finite element method for parabolic equations: (I) a posteriori estimation, Numer. Math. 40 (1982), 339-371. MR 85d:65052a

3. M. Bieterman and I. Babuška, The finite element method for parabolic equations: (II) a posteriori estimation and adaptive approach, Numer. Math. 40 (1982), 373-406. MR 85d:65052b

4. I. Babuška and C. Rheinboldt, Error estimates for adaptive finite element computations, SIAM J. Numer. Anal. 15 (1978), 736-754. MR 58:3400

5. Z. Chen and S. Dai, Adaptive Galerkin methods with error control for a dynamical GinzburgLandau model in superconductivity, SIAM J. Numer. Anal. 38 (2001), 1961-1985. MR 2002g:65114

6. Z. Chen and S. Dai, On the efficiency of adaptive finite element methods for elliptic problems with discontinuous coefficients, SIAM J. Sci. Comput. 24 (2002), 443-462.

7. Z. Chen, R.H. Nochetto and A. Schmidt, A characteristic Galerkin method with adaptive error control for continuous casting problem, Comput. Methods Appl. Mech. Engrg. 189 (2000), 249-276. MR 2001f:80003

8. Z. Chen, R.H. Nochetto and A. Schmidt, Error control and adaptivity for a phase relaxation model, Math. Model. Numer. Anal. 34 (2000), 775-797. MR 2001i:65117

9. P.G. Ciarlet, The Finite Element Method for Elliptic Problems, North-Holland, Amsterdam, 1978. MR 58:25001

10. Ph. Clément, Approximation by finite element functions using local regularization, RAIRO Anal. Numer. 9 (1975), 77-84. MR 53:4569

11. W. Dörfler, A convergent adaptive algorithm for Possion's equations, SIAM J. Numer. Anal. 33 (1996), 1106-1124. MR 97e:65139

12. K. Eriksson and C. Johnson, Adaptive finite element methods for parabolic problems I: A linear model problem, SIAM J. Numer. Anal. 28 (1991), 43-77. MR 91m:65274

13. K. Eriksson and C. Johnson, Adaptive finite element methods for parabolic problems IV: Nonlinear problems, SIAM J. Numer. Anal. 32 (1995), pp. 1729-1749. MR 96i:65081

14. P.K. Moore, A posteriori error estimation with finite element semi- and fully discrete methods for nonlinear parabolic equations in one space dimension, SIAM J. Numer. Anal. 31 (1994), 149-169. MR 94m:65145

15. P. Morin, R.H. Nochetto and K.G. Siebert, Data oscillation and convergence of adaptive FEM, SIAM J. Numer. Anal. 38 (2000), 466-488. MR 2001g:65157

16. R.H. Nochetto, A. Schmidt and C. Verdi, A posteriori error estimation and adaptivity for degenerate parabolic problems, Math. Comp. 69 (2000), 1-24. MR 2000i:65136 
17. M. Picasso, Adaptive finite elements for a linear parabolic problem, Comput. Methods Appl. Mech. Engrg. 167 (1998), 223-237. MR 2000b:65188

18. A. Schmidt and K.G. Siebert, ALBERT: An adaptive hierarchical finite element toolbox, IAM, University of Freiburg, 2000. http://www.mathematik.uni-freiburg.de/IAM/ Research/projectsdz/albert.

19. R. Verfürth, A Review of A Posteriori Error Estimation and Adaptive Mesh Refinement Techniques, Teubner (1996).

20. R. Verfürth, A posteriori error estimates for nonlinear problems: $L^{p, r}\left(0, T ; W^{1, \rho}(\Omega)\right.$ Finite element discretization of parabolic equations, Numerical Methods in Partial Differential Equations 14 (1998), 487-518. MR 99g:65099

LSEC, Institute of Computational Mathematics, Academy of Mathematics and System Sciences, Chinese Academy of Sciences, Beijing 100080, Peoples Republic of China

E-mail address: zmchen@lsec.cc.ac.cn

Institute of Computational Mathematics, Academy of Mathematics and System Sciences, Chinese Academy of Sciences, P.O. Box 2719, Beijing 100080, Peoples Republic of CHINA

E-mail address: fjia@lsec.cc.ac.cn 